\title{
Analysis and Evaluation of Indian Industrial System Requirements and Barriers Affect During Implementation of Industry 4.0 Technologies
}

ABINASH JENA ( $\square$ abinashjena741@gmail.com )

National Institute of Technology Rourkela https://orcid.org/0000-0001-7292-813X

Saroj Kumar Patel

National Institute of Technology

\section{Research Article}

Keywords: Industry 4.0, Fuzzy, System Requirements, Barriers, Correlation

Posted Date: June 22nd, 2021

DOI: https://doi.org/10.21203/rs.3.rs-603058/v1

License: (c) (1) This work is licensed under a Creative Commons Attribution 4.0 International License.

Read Full License

Version of Record: A version of this preprint was published at The International Journal of Advanced Manufacturing Technology on February 19th, 2022. See the published version at https://doi.org/10.1007/s00170-022-08821-0. 


\title{
Title: 'Analysis and Evaluation of Indian Industrial System Requirements and Barriers Affect During Implementation of Industry 4.0 Technologies'
}

\author{
Abinash Jena ${ }^{1}$ and Saroj Kumar Patel ${ }^{2}$
}

1. (Corresponding Author) Research Scholar, Department of Mechanical Engineering, National Institute of Technology, Rourkela, Email: abinashjena741@gmail.com, abinash jena@nitrkl.ac.in ;

2. Professor, Department of Mechanical Engineering, National Institute of Technology, Rourkela, Email: skpatel@nitrkl.ac.in

\section{ABSTRACT}

In recent years, competition among the Indian Manufacturing Industries (IMI) has increased enormously in the global market. The current uncertainty in the market context is characterised and governed by the customised requirements of the customers. Thus, the manufacturing system in the industries should be capable of adapting the parameters like flexibility in scalability, variety, agility, system responsiveness, inter-connectivity, automatic data exchange with communication among the manufacturing systems, transparency and human-machine interaction, which are the main components and principles of Industry 4.0 (14.0). Thus, adopting 14.0 plays a vital role to corroborate and its long-term survival in the global marketplace. However, very few research work considerations contribute to the issues induced during the adoption of 14.0 in manufacturing industries. This paper aims to minimise the gap between the existing Industrial System Requirements (ISR) and the challenges faced during the implementation of 14.0 technologies in existing Industries. The identified ISR and barriers were evaluated and analysed based on the data set collected from a questionnaire-based survey. Fuzzy multi-criteria analysis is conducted to identify the most weighted $S R$ and barriers and ranked them concerning their importance. Furthermore, the inter-item correlation between both of them is analysed. This research work offers the researchers, practitioners, and industrialists an opportunity to formulate MCDM problems through numerous case studies, prioritising the top barriers and system requirements and the interrelationship shared between them.

\section{Keywords: Industry 4.0, Fuzzy, System Requirements, Barriers, Correlation}

\section{INTRODUCTION}

Today the survival of a manufacturing industry depends on its flexibility, agility, quick responsiveness to fluctuating market demand, scalability, variability, mass customisation as per the preference of the customer, excellent quality and post-sale service (Hu et al., 2008; Lotter \& Wiendahl, 2008; Wang \& Koren, 2012; Pattanaik \& Jena, 2019). These factors are driven by data sharing and monitoring, data transparency and connectivity among the manufacturing systems without any barriers (Salam, 2019). 'Industrie 4.0' (14.0) or the Fourth Industrial Revolution is the integration and interconnection of Machines and Robots, Sensors and Actuators, Basic Physical and Software Systems, Interoperability, Logistics and Storage Systems, Real-Time Data Transfer and Data Transparency, Customers and People, Man to Machine Interaction, Market and Other Economic Sectors, Standardisation of Production Process, Information and Communication Technologies, Cyber-Physical Systems, Internet of Things, Big data and Cloud Computing which are interconnected among themselves and communicate with one other in their social communication platform with the help of wired or wireless networks (Gilchrist, 2016; Kamble et al., 2018; Li et al., 2018; Calia \& D'Aprile, 2020; Matt \& Rauch, 2020). It integrates the feedback loop 
from the machines, systems, sensors, clouds storage, inventory stock, customers, workers, employees, logistics, management, market demand with each other with the support of internet connectivity to monitor the production, sales and services continuously as well as storing the data for continuous improvement in productivity (Ustundag \& Cevikcan, 2018).

In the emerging global economy, for the long-term survival of the manufacturing industries to remain intact in the global market competition, there is a need for serious upgradation of the existing manufacturing system to the smart factory that is the fourth industrial revolution. As of now, the 14.0 technologies are still in the research and development phase, mostly confined to the implementation phase, as specific unavoidable driving barriers affect during adoption in the current manufacturing industries (Frank et al., 2018; Kamble et al., 2018; Luthra \& Mangla, 2018; Sanders et al., 2016).

Few papers point out the barriers that affect the implementation of 14.0 technologies in existing industries. However, the literature is silent on addressing the gap between the requirements of existing Indian Industries in the context of 14.0 and the issues caused while implementing 14.0 technologies. As a result, research interest grew in the 14.0 implementation strategy. This paper explores and identifies various current industrial system requirements (ISR) and the barriers to implement 14.0 in existing manufacturing systems from the literature review and interaction with numerous industry experts. First, the existing ISR in Indian Manufacturing Industries (IMI) was identified through an exhaustive literature review and finalised thirteen requirements (such as Reducing cost, increasing flexibility in scalability and variability, mass customization etc., elaborated in section 5.1) by interviewing and opinions of the Industrial experts.

Similarly, in the next phase, the implementation issues or the implementing barriers faced by IMI (such as inadequate digital culture, implementation cost, etc.) were identified through the literature review. With a lengthy discussion, many industry experts and academicians currently working in smart factory technologies, from their opinions, eighteen barriers that affect 14.0 implementation were finalised.

Then for quantifying and evaluating the identified ISR and barriers, a questionnaire survey was conducted where the expert's opinion and ratings are collected, especially those working on 14.0 and its core technologies in industries, research organisations, and academicians. Then the collected linguistic data were analysed and classified into barriers and adaptation factors. This paper analyses the collected data set to identify the most weighted ISR factors and barriers that affect the implementation of 14.0 through evaluation. It is obtained from the fuzzy multi-criteria analysis. Next, correlation analysis between these ISR and the barriers of 14.0 is obtained, which determines the relationship between them. It was obtained and analysed by IBM SPSS software version 25.

The structural layout of this paper is organised as follows. Theoretical background about 14.0, some of its core components and the Existing Indian manufacturing scenario in the context of 14.0 are presented in Section 2 and 3, respectively. The literature survey related to this work is given in Section 2. The research methodology is presented in Section 3. Data analysis and evaluation are presented in Section 4. Results and discussions are presented in Section 5. Conclusion and future works are presented in Section 6.

\section{THEORETICAL BACKGROUND}

The first industrial revolution began at the end of the 18th century, represented by mechanical production plants based on water and steam power, in which the products were made in a family workshop by craftsmen and their apprentices; the second industrial revolution started at the beginning of the 20th century which brought a paradigm of mass labour production based on electrical energy to produce a large volume of standardised products with division of labour and 
specialised skills at a low unit cost; the third industrial revolution began in the 1970s with the characteristics of automation based on electronics, computers applications and information technology, where the manufacturing evolved from mass production to mass customisation, with the help of programmable machines to produce standardised products with some degree of flexibility at the production line and sub-assembly or final assembly (Zhou et al., 2016; Santos et al., 2017; Rojko, 2017; Laudante, 2017; Moktadir et al., 2018; Dean \& Spoehr, 2018; Ustundag \& Cevikcan, 2018; Fatorachian \& Kazemi, 2018; Iyer, 2018; Lasi \& Kemper, 2019; Moeuf et al., 2019); and the fourth industrial revolution which is Industry 4.0 has emerged recently over the last few years (lyer, 2018; Lu, 2017; Xu et al., 2018). It has emerged as a promising technology framework that can be used for integrating and extending manufacturing processes at both intra-organisational and interorganisational levels where machine to machine communication, real-time data transparency, human to machine interaction with the help of information and communication technology (ICT), Internet of things (IoT), smart factory, cyber-physical systems (CPS) and cloud computing is integrated for manufacturing. The core concept of Industry 4.0 is the integration of the basic physical system and the software system, integration with other branches and economic sectors, integration with other industries and industry types (Li, 2018). Industry 4.0, otherwise known as smart manufacturing and cognitive manufacturing, offers new manufacturing firms opportunities to analyse and use design, production, sourcing, and inventory data to realise their modernisation vision (Yin et al., 2018).

In 14.0, the entire industrial production system is transformed and digitalised by merging the traditional production process with the internet and information and communication technologies (ICT) infrastructures (Cohen et al., 2019). In 14.0, the Industrial loT (IloT) is the primary tool that applies analytical models and data science to analyse real-time data sharing, real-time monitoring, also uses smart devices to sense, capture, measure and transfer data from multiple machines, manufacturing processes and its systems and finally reconfigure and automates the manufacturing systems as per the requirement of the customer (Da Xu et al., 2018). It combines the data from intelligent sensors with artificial intelligence and performs data analytics, optimising the manufacturing process in real-time (Lu, 2017). It consists of Industrial Wireless Networks (IWN), internet, machines, equipment, networks, cloud storage and computing. In this system, the customers can customise and personalise their products to be manufactured from its end. The customised data are transmitted to the industrial cloud and manufacturing plant via wired or wireless networks. Based on these received data, the manufacturer integrates the design for the product and then optimises production and simulates it. It also manages and monitors the production process and diagnosis to produce the required products efficiently. With self-optimisation and self-directed decision-making, machines, cloud computing, and equipment adapt and reconfigure to improve performance (Roblek et al., 2016; Wang et al., 2016; Schmidt et al., 2015).

In 14.0, Cyber-Physical System (CPS) is an entirely data-driven application that inter-connects the virtual space with physical components systems through integrated computing, complex algorithms, sensors data, embedded systems, communication, data analytics and storage capabilities with the help of complex networks and Internet (Cheng et al., 2016; Gilchrist, et al., 2016). It collaborates planning, analysis, modelling, design and implementation, and maintenance in the manufacturing process more securely with information exchange among embedded computer systems. CPS maximises efficiency and industrial growth, modifies workforce performance, minimises errors, and produces higher-quality products with lower costs. Interoperability is the critical factor of Industry 4.0 , enabling the two systems or machines to communicate, cooperate and understand each other, make decisions by exchanging data and sharing information and knowledge without human interference (Lu, 2017). 


\section{INDIAN MANUFACTURING INDUSTRIES AND INDUSTRY 4.0}

At present, the concept of Industry 4.0 is still in the developing stage in India. It needs proper empirical research and analysis, awareness and practice. The statistical data have been surveyed and reviewed through various sources from government websites, world bank, government financial reports, yearly manufacturing reports, Indian brand equity foundation reports and various news articles to analyse India's present manufacturing scenario, its performance, capacity utilisation, exports, growing demand and measures to meet the demand of the customer.

Recently, India's manufacturing sector has witnessed strong growth over the past few years, as the estimated gross value added is up to US\$ 390.43 Billion and projected to grow more up to USD\$ 398 billion (IBEF, 2016). It is observed that the growth of the manufacturing sector of India weighs 77.63 per cent in the Index of Industrial Production (IIP) and dominating to no small extent to that of mining and electricity production ("MSPI," 2019). This not only shows the production levels of the industries but also specifies the potential for future investments. As per the latest review, capacity utilisation in India's manufacturing sector is around 74.8 per cent in the second quarter of 20182019. In the same period, the average new demands of customers also increased as the manufacturing units grew 26.1 per cent compared to the previous years. India's Merchandise export performance witnessed a 9.52 per cent growth to reach US\$271 billion. It also reflects India's emergence to compete with other countries worldwide market scenario (IBEF, 2019).

These reviews based on market context and uncertainty in global performance, the fastest emerging economy motivate the research work to use it to adapt 14.0 in existing manufacturing industries. Today the leading countries have already geared up and started implementing advanced technologies and Internet and Information Technology on a large scale in their existing systems. India is majorly focusing on implanting its new policies and improving infrastructure (Grant Thornton Report, 2017).

At present, industry 3.0 is not entirely implemented throughout the manufacturing sector of India as per the industrial reports from an audit and advisory firm KPMG in association with All India Management Association, and many sectors, especially the SMEs, are still in post electrification phase, that uses basic machinery systems for production and lacks core technology. India's Existing manufacturing industry is still a resource consumption type, has labour-intensive industries and resides at the industrial chain's low end. The integration of physical systems on cyber platforms, the basic premise of 14.0, is still at its early stages (AIMA-KPMG Report, 2018).

The Government of India policies is continuously pushing to adopt modern technology and digital transformation by collaborating with more FDI for domestic production. The national manufacturing policy also predicts that the GDP share in manufacturing increases to 25 per cent by 2021 (IBEF, 2019). However, there is an increment in R\&D funding, not compared to match to developed countries (United States, Germany and Japan) (Agarwal, 2019; Samarth Udyog, 2018). Policies like Samarth Udyog Bharat 4.0, an Industry 4.0 initiative for IMI launched by the Ministry of Heavy Industry and Public Enterprises, Smart Cities projects enabling the Internet of Things Platform, and Digital India policies in 2018 were formed and governed research and development in existing manufacturing environments. The rate of adoption of 14.0 are expected to be highest in Industries such as Utilities, Manufacturing, Automotive and Transportation \& Logistics are expected (Petit et al., 2019).

\section{LITERATURE REVIEW}

'Industrie 4.0' also known as 'Industry 4.0' (14.0) or the Fourth Industrial Revolution, was initially introduced during the Hannover Fair in 2011 and was officially declared in 2013. It is a German strategic initiative to take a ground-breaking role in industries that are currently revolutionising the 
manufacturing sector by integrating with modern technologies and connectivity and quick adaptability of manufacturing systems to provide maximum output as per the demand with efficient utilisation of resources along with maintaining better product quality (Kamble et al., 2018). Since then, numerous research has been conducted in recent years on loT, Artificial Intelligence (AI), CPS, Cyber Security, Universal Standardization, Horizontal and Vertical Integration, ICT, Cloud Computing, Man-Machine Interactions integration into 14.0 systems. Nevertheless, major research works are yet to be done on Industrial integration, Data Privacy, Implementing Strategy, Empirical validation and testing of the 14.0 technologies, real-time studies on ERP, Smart Devices, Smart Sensors, Reconfiguring tools and equipment, Machine-Machine communications, BlockChain, CPS, Data Science and Data Analytics (Liao et al., 2017; Mueller et al., 2017; Pereira \& Romero, 2017; Ghobakhloo, 2018; Kamble et al., 2018; Li et al., 2018; Pico \& Holgado, 2018; Xu et al., 2018). Although the literature survey in 14.0 and its technologies is quite vast and exhaustive, this paper's literature survey is circumscribed to our core area of research interest. Identifying the existing ISR and the relevant barriers of 14.0 implementations is a crucial task. In this literature, we reviewed the recent progress in identifying the challenges to adopt 14.0 and its implementation and its related publications from the last five years.

(Zhou et al., 2016) addressed various challenges for implementing 14.0 paradigms, such as the development of smart devices, the construction of the network environment, big data analysis and processing, and digital production, are also identified and also proposed strategic planning includes constructing a CPS network and discussing two significant themes based on the smart factory and intelligent production. (Lu, 2017) identifies and presents the critical issue and conceptual framework of the interoperability and various research challenges of Industry 4.0 and its implementation. Potential barriers to adapt industry 4.0 are identified and analysed by (Kamble, 2018); the relationships among them were developed using interpretive structural modelling (ISM) and fuzzy MICMAC methodology in the Indian manufacturing context with the help of experts. This work helps identify and classify the significant barriers, revealing each identified barrier's direct and indirect effects on the 14.0 adoption. Various challenges to adopt 14.0 technologies in IMI were identified by (Luthra \& Mangla, 2018). These were evaluated and prioritised potential challenges for supply chain sustainability through survey and ratings from the experts and analysed through Explanatory Factor analysis and Analytical Hierarchy Process tools. It also helps the industrialists incorporate environmental protection and directing to eliminate the potential challenges in adopting 14.0 technologies for the sustainability of the supply chain. Several challenges for implementing 14.0 were identified and evaluated using the best-worst method, a multi-criteria decision-making method (Moktadir et al., 2018) to identify the most weighted challenges and ranked in decreasing order along with a case study. This results in addressing these challenges for the industrialists and practitioners to build up specific strategies for implementing 14.0 technologies.

The research objective is quite clear from the above literature and confined to implementing barriers from another perspective, which needs more empirical evaluation and analysis. Most of the findings are confined to identify the challenges faced during the implementation through surveys and validating using various research tools.

Strategic decisions were suggested by (Zhang et al., 2016) to adopt German "I4.0" in Chinese manufacturing industries, which provided a path for advanced manufacturing industrial development with clear goals and practical measures. Suggestions were provided to materialise the transformation and upgradation of the present manufacturing scenario to 14.0. The relationship between lean production practices and the implementation of Industry 4.0 is examined by (Tortorella \& Fettermann, 2018), in which relevant data were collected through a questionnaire survey from various industries. Clustering tools are used to find the relationship within the database, LP practices and 14.0. (Kamble et al., 2019) investigates the direct and indirect effect of 14.0 technologies, lean manufacturing practice, sustainable organisational performance, and the relationship between them 
based on survey and data collected from respondents. This paper also provides empirical validation, which is a critical factor for the implication of 14.0 technologies in existing manufacturing industries.

The above literature points out the implementation strategy, inter-item relationship, effects of 14.0 technologies with different manufacturing practices. But the literature is silent on how ISR is affected by barriers caused while implementing 14.0 technologies. More research work needs to be addressed to connect the bridge and the relationship between the ISR and the barriers affecting the implementation of 14.0 technologies.

The system requirement factors of 14.0 and implementation barriers, finding the highest weighted among them and the inter-relationship shared among them with the application of fuzzy multi-criteria decision analysis and inter-item co-relation analysis, respectively, is the unique findings in this paper. The concept is novel and needs extensive understanding from the theoretical, practical and managerial aspects of the ethical implications of approaching 14.0 technologies in existing manufacturing industries. There's a need to adapt and determine the customer's fluctuating demands, inter-connectivity, mass customisation, transparency, and agility in the production process.

\section{RESEARCH METHODOLOGY}

This section of the paper identifies the factors, questionnaire development and collection of data through a survey. At first, the existing Industrial System Requirements (ISR) and Barriers to adapt Industry 4.0 (14.0) technologies in Indian Manufacturing Industries (IMI) are identified and finalised. A survey-based approach is considered, where the industrial experts provide their valuable ratings and feedbacks as per the designed questionnaire. The ratings are collected as a linguistic database where further analysis and evaluation of the identified ISR and Barriers are carried out in the next section.

The following research works that were performed are presented in the subsequent subsection.

\subsection{IDENTIFICATION OF EXISTING ISR}

The following thirteen crucial ISR were identified and finalised from the literature review and interviewing industrial experts from several IMI. These factors are the core parameters of Industry 4.0, and its application is highly required in existing IMI (Shrouf et al., 2014; Gilchrist et al., 2016; Oesterreich \& Teuteberg, 2016; Sanders et al., 2016; S. Wang et al., 2016; J. Xu et al., 2016; Rojko, 2017; Mueller et al., 2017; Sevinç et al., 2018; Fettermann et al., 2018; Frank et al., 2018; Telukdarie et al., 2018; Ustundag \& Cevikcan, 2018; Sony \& Naik, 2019). These ISR are the critical elements to improve productivity, implement innovative ideas, access and analyse the performance measure, and eliminate complexities virtually before final implementation. The industries need to adapt these following system requirement factors for their long-term survival and compete in the global market:

1. Reducing cost (A 1)

2. Increase flexibility in scalability and variety (A 2)

3. Increasing system responsiveness (A 3)

4. Improving inter-connectivity among all the systems (A 4)

5. Automatic data exchange and communication (A 5)

6. Transparency through real-time data monitoring and exchange (A 6)

7. Human-machine interaction (A 7)

8. Mass customisation (A 8)

9. Achieving greater efficiency (A 9)

10. Improving productivity (A 10)

11. Higher resource utilisation ( $A$ 11)

12. Increasing global business (A 12)

13. Reducing the lead time of production (A 13) 


\subsection{IDENTIFICATION OF BARRIERS TO ADAPT INDUSTRY 4.0}

After going through the various research publications papers, related published books and interviewing experts for their valuable inputs, the following eighteen barriers have been identified and finalised. The following identified factors are the challenges faced during implementing Industry 4.0 in IMI ( Lotter \& Wiendahl, 2008; Sanders et al., 2016; Iglesias-Urkia et al., 2017; Mueller et al., 2017; Kamble et al., 2018; Li et al., 2018; Luthra \& Mangla, 2018; Moktadir et al., 2018; Frank et al., 2018; Petit et al., 2019; Kamble et al., 2019; Singla et al., 2019).

1. Lack of understanding and knowledge $(\mathrm{C} 1)$

2. Inadequate digital culture $(\mathrm{C} 2)$

3. Employee flexibility required to learn and adapt (C 3)

4. Implementation cost (C 4)

5. Virtualisation and dynamic Integration (C 5)

6. Integration of physical systems with cyber systems $(C 6)$

7. Lack of proper global standards and reference architecture (C 7)

8. Uniform standards for tools \& equipment, systems, languages, networks, data services and reference architecture like design and selection (C 8)

9. Quick reconfiguration of manufacturing systems, assembly systems, inspection and material handling systems (C 9)

10. Real-time full data sharing and monitoring among various units, customers, suppliers, logistics and a few selected data sharing with other industries $(C 10)$

11. Government policies and support (C 11)

12. Data and Information security (C 12)

13. Privacy protection (C 13)

14. Clearly defined investment returns and economic benefits of digital investment $(C 14)$

15. Uncertainty in the interests of industry 4.0 (C 15)

16. Several complex legal issues (C 16)

17. Lack of Integration of technology (C 17)

18. Data quality and big data (C 18)

Brief descriptions of the identified ISR and barriers are presented in Table 1.

\subsection{QUESTIONNAIRE DEVELOPMENT}

A set of elaborate questionnaires was designed initially as per the ISR and barriers that affect industry 4.0 implementation in IMI. Taking two types of rating using five-point Likert scale which varies as per the question (for example, ratings for the first scale ranging very low (VL), low (L), medium $(\mathrm{M})$, high $(\mathrm{H})$ and very high $(\mathrm{VH})$ and the second scale ranging strongly disagree (1), disagree (2), neither agree nor disagree (3), agree (4) and strongly agree (5) as shown in Table 2. A five-point Likert scale was used to increase the responses rate and response quality and helps the respondents to respond to the questionnaire with ease. As the response data obtained is highly uncertain, the best mathematical simplification and approximation of such complex functions of the data set is Trapezoidal Fuzzy functions (Zhang et al. 2005). Thus, Trapezoidal fuzzy numbers were considered in this methodology according to which the questionnaire is designed for the survey. Later on, the questionnaire was modified and tweaked as per the data's requirements, such as to receive qualitative data with less ambiguity for accurate analytical results. Finally, a set of forty-six questions were designed, structured and finalised for the survey, which would take about twenty minutes for the experts to answers the questions. Various extra questions were added about the respondents about their work experience, department, sector, educational qualifications, organisation name and any remark they wanted to provide along with the survey.

The respondents were also asked some other questions like how much they are aware of Industry 4.0, its core technologies, and the first thing that comes to mind upon hearing about its core technologies and implementation. Questions related to sectors in an organisation that could be 
benefitted from the application of Industry 4.0 and how much they believe that the factors of Industry 4.0 would help their organisation.

It was focused mainly on the following factors that are considered as systems requirements of Industry 4.0; Reducing cost, Increase flexibility in scalability and variety, Increasing system responsiveness, Improving inter-connectivity among all the systems, Automatic data exchange and communication, transparency through real-time data monitoring and exchange, Human-machine interaction, Mass customisation, Achieving greater efficiency, Improving productivity, Higher resource utilisation, Increasing global business and Reducing the lead time of production. Some questions were made mandatory without which the respondent could not submit the form, which eradicates the risk of incomplete data sets; hence all the questionnaire can be used for analysis (Kamble et al., 2019). Participation in this survey was kept voluntary to the respondents instead of mandatory.

\subsection{SURVEY AND DATA COLLECTION}

For conducting the survey, the link to the survey form was initially sent to more than three hundred respondents through Emails, Linkedln direct message, text message, social platforms, and personal contacts in the first month. At first, the survey was confined to the manufacturing industries across major cities in India. Then it was expanded to service-based and solution-based industries and finally to all types of organisations. One cover letter was attached with the form and which mentions the respondents to fill it up only if they have the knowledge or working in Industry 4.0 technologies. The respondents were also given a limited time frame of about five weeks to submit their response. From the initial survey, only 30 complete responses were received after the end of one month. The pilot Reliability test for the first ten then thirty were analysed in SPSS for the received data to check whether the questionnaire set is applicable and robust for surveying the next phase or not. The Cronbach's Alpha scored 0.90 and 0.91 , respectively, which is more than 0.70 and is sufficient to carry on with the survey without any modification of the questionnaire (Kamble et al., 2018).

After getting a satisfactory result, more than seventy per cent next phase of the survey was conducted. In the next month, two hundred respondents were sent through secondary sources such as Industrial websites, Industry 4.0 forums, institute alumni, google searches, industrial sites and articles, newspaper articles and other sources. Then in the next month, the survey was sent to subsequent four hundred respondents, counting fifty respondents each week for the next two months through the same medium and telephonic interview.

A week later, multiple remainder emails, telephonic calls, and text messages were sent to the rest of them. After numerous reminders and discussions about this survey with some respondents, a total of 224 responses was received finally at the end of six months. The response rate is 24.8 per cent which is sufficient to analyse the research work. All these responses were structured, categorised and noted into an excel sheet database for each respondent separately (Malhotra \& Grover, 1998).

The respondents were from various departments and designations so that there shouldn't be any biases in the survey, such as working as in Sales, CEO, Assistant Professor, Consultant, Director, Manager, Data Scientist, Design and Release Engineer, Senior Analyst, Marketing strategist, HR and Marketing, Lead Mechanical Engineer, Procurement Manager, Senior Engineer, Automation Technologist, Co-founder, Digital Manufacturing - System Specialist, Technical Engineer of Projects, Manager of Industry Automation Team, Presales \& Manager, Digital Domain Lead Consultant, Software Engineer and R\&D Analyst.

\section{DATA ANALYSIS AND DISCUSSION}

In this section, the pilot data analysis is conducted, and necessary tests like KMO and Bartlett's were run, which are prerequisites for validating the data set and checking the legitimacy of the identified factors. Then Descriptive statistics analysis is conducted to check the significance of the 
identified system requirement factors and barriers. Then Fuzzy techniques used to evaluate and prioritise the identified ISR and the barriers. Then, and finally, inter-item correlation analysis is conducted among them.

\subsection{Initial Statistical Analysis}

The statistical data have been initially surveyed and collected through various sources from government websites, world bank, government financial reports, yearly manufacturing reports, Indian brand equity foundation reports and various news articles to analyse the modern manufacturing environment, its performance, capacity utilisation, exports, growing demand and measures to meet the demand of the customer. These data help determine the decisions and remarks for the market context's current uncertainty based on the customers' demands and variable production.

The next step, the collected data's pilot analysis, was conducted to know about the respondent's background, educational qualification, working experience, and industrial background. Fig. 1 shows the survey's timeline and the responses received from the respondents from the various sectors. Fig. 2 shows the type of organisation to which the respondents belong. It characterises the type of industries in which the respondents are currently working. About seventy-six per cent of the respondents were from various private sectors and MNCs throughout the country, and little more than eight per cent were from educational and public sectors combined. Most of the respondents are from product-based sectors that are about twenty-nine per cent, the next twenty-five per cent are from service-based sectors, and the rest belongs to IT, R\&D, educational sectors. Fig 3 . This shows how much the respondents are aware of 14.0 and its core technologies. It is observed that the maximum number of respondents to a total of about sixty-seven per cent of them are highly aware, and about twenty-seven per cent of them are somewhat aware and very few of them are less aware of 14.0 technologies. Fig. 4 shows the respondent's educational background. It is observed that the maximum number of respondents are about fifty-three per cent are postgraduates, and thirty-nine per cent are graduates. Fig. 5 represents the respondent's years of working experience ranging from one year to fifty years. From the pilot analysis, it is observed that most of the respondents belonging to private sectors and MNCs are currently working on I 4.0 and its core technologies.

\subsection{Necessity Analysis}

The following necessity analysis was conducted using IBM SPSS 25 to validate the legitimacy of the collected data. Any future analysis and evaluation can be conducted, relying on this data set. The whole data set's reliability coefficient test was performed to check the survey's data set's ambiguity, clarity, and relevance. Table 3 shows the Cronbach alpha values score to 0.88 (above 0.70 ), ensuring the survey data's appropriateness and can be analysed further without modification and reframing the questionnaire (George \& Mallery, 2018 ).

Kaiser-Meyer-Olkin (KMO) analysis is performed to measure the proportion of variance in factors identified and quantified for analysis due to the underlying factors' presence. The KMO proportion value must be lower (Robert and Popović, 2015; George \& Mallery, 2018; S. Kamble et al., 2019b; Luthra \& Mangla, 2018). Table 3 shows that the KMO proportion obtained is 0.872 , greater than 0.5 and is considered meritorious for factor analysis and the identified factors are legit.

Bartlett's Test of Sphericity is also analysed to identify the identified barriers' correlation matrix and the ISR. We obtained the result for these identified factors, whose significant value is significantly less, that is $(p<0.01)$, hence factor analysis can be used in our data set in future works (Meyers et al., 2013; Field, 2017; Luthra \& Mangla, 2018). 
Descriptive statistics analysis was carried out for both barriers and ISR and tabulated and plotted in Fig 9 and Fig 10. It is observed that the resulting mean value for both barriers and ISR stands out to be higher than ' 3 '. Thus it is concluded that the data set collected are significant and can be used for various future analysis (Field 2017; Luthra \& Mangla, 2018).

\subsection{Multi-Criteria Analysis}

As the current Indian manufacturers, the ISR and the challenges generated by barriers to adapt Industry 4.0, the respondents believe these factors would play a vital role in their respective organisation (Moktadir et al., 2018). The identified factors are rated and evaluated to identify the most dominant barrier that affects Industry 4.0 in their organisation and identify the most dominant existing ISR that the respondents believe would benefit their organisation. As these several barriers and ISR may contain several conflicting criteria, and the ratings from the respondents are subjective, therefore to reduce the effect of vagueness in the feedback and to narrow down the selection process fuzzy multi-criteria decision analysis tool is implemented for obtaining the weightage of each criterion (Zhang et al., 2005). This weightage leads to identify the most dominant factor.

As shown in Figure 6, five fuzzy sets are used to collect the ratings from the respondents through the questionnaires in the linguistic terms of $\mathrm{VL}, \mathrm{L}, \mathrm{M}, \mathrm{H}$ and $\mathrm{VH}$ for very low, low, medium, high and very high, respectively as well as 1,2, 3, 4 and 5 for strongly disagree, disagree, neither agree nor disagree, agree and strongly agree respectively. All the rating was collected and categorised for efficient use of the data for analysis. These five fuzzy sets are numerically expressed as trapezoidal fuzzy numbers (TrFNs), as produced in Table 2.

At first the linguistic terms for each respondent are converted into fuzzy ratings. For example, Respondent 1(k=1), provided the ratings for each barriers $C_{j 1}$ as, 4, 4, 4, 3, 3, 5, 4, 4, 5, 5, 3, 3, 2, $3,4,2,4$ and 4 . This is represented by the set in fuzzy ratings such as, $\left(\begin{array}{llll}0.5 & 0.6 & 0.7 & 0.8\end{array}\right),\left(\begin{array}{ll}0.5 & 0.6\end{array}\right.$ $\left.\begin{array}{ll}0.7 & 0.8\end{array}\right),\left(\begin{array}{llll}0.5 & 0.6 & 0.7 & 0.8\end{array}\right),\left(\begin{array}{llll}0.4 & 0.5 & 0.5 & 0.6\end{array}\right),\left(\begin{array}{lllll}0.4 & 0.5 & 0.5 & 0.6\end{array}\right),\left(\begin{array}{llll}0.7 & 0.8 & 0.9 & 1.0\end{array}\right),\left(\begin{array}{lll}0.5 & 0.6 & 0.7\end{array}\right.$

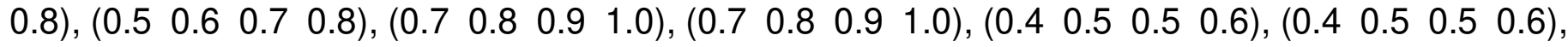
$\left(\begin{array}{llll}0.2 & 0.3 & 0.4 & 0.5\end{array}\right),\left(\begin{array}{llll}0.4 & 0.5 & 0.5 & 0.6\end{array}\right),\left(\begin{array}{llll}0.5 & 0.6 & 0.7 & 0.8\end{array}\right),\left(\begin{array}{llll}0.2 & 0.3 & 0.4 & 0.5\end{array}\right),\left(\begin{array}{llll}0.5 & 0.6 & 0.7 & 0.8\end{array}\right)$ and $\left(\begin{array}{llll}0.5 & 0.6 & 0.7 & 0.8\end{array}\right)$ respectively.

Similarly, all the ISR' ratings are converted into fuzzy numbers, generating a fuzzy matrix. The ratings are aggregated by the following mathematical expressions for computing-the aggregate fuzzy weights for both barriers to adapt (C) and implementation factor $(A)$ for calculating this data.

\section{Aggregated fuzzy weights and Defuzzification}

The set of ' $\mathrm{k}$ ' respondents is represented as $P_{k}=\left(P_{1}, P_{2}, P_{3}, \ldots, P_{k}\right)$, where $k=1$ to 224 . The set of ' $\mathrm{j}$ ' barriers and ' $m$ ' ISR are represented as $C_{j}=\left(C_{1}, C_{2}, C_{3}, \ldots, C_{j}\right)$ and $A_{m}=\left(A_{1}, A_{2}, A_{3}, \ldots, A_{m}\right)$ where, $j=1$ to 18 and $m=1$ to 13 . As per the $k^{\text {th }}$ respondent's ratings, the fuzzy rating for the $j^{\text {th }}$ barriers' weights to adapt industry 4.0 is represented as $W_{j k}=\left(W_{j k 1}, W_{j k 2}, W_{j k 3}, \ldots, W_{j k 4}\right)$. Similarly, The fuzzy rating for the weights of the $m^{\text {th }}$ ISR of industry 4.0, as per the ratings given by the $k^{\text {th }}$ respondent, is represented as $W_{m k}=\left(W_{m k 1}, W_{m k 2}, W_{m k 3}, \ldots, W_{m k 4}\right)$ ( Zhang et al. 2005; Wang, 2006; Liu \& Jin, 2012; Zhang et al., 2013; Musani \& Jemain, 2017). The aggregated fuzzy weights $\left(W_{j k}\right)$ concerning each barrier is calculated as $\left(W_{j k 1}, W_{j k 2}, W_{j k 3}, \ldots, W_{j k 4}\right)$ such that $j=1,2, \ldots 18$ and $k=1,2, \ldots 224$ and

$$
\begin{aligned}
& W_{j k 1}=\min _{k}\left\{W_{j k 1}\right\} \\
& W_{j k 2}=\frac{1}{k} \sum_{k=1}^{K} W_{j k 2} \\
& W_{j k 3}=\frac{1}{k} \sum_{k=1}^{K} W_{j k 3} \\
& W_{j k 4}=\max _{k}\left\{W_{j k 4}\right\}
\end{aligned}
$$

The aggregated fuzzy weights $\left(W_{m k}\right)$ with respect to each ISR is calculated as $\left(W_{m k 1}, W_{m k 2}, W_{m k 3}\right.$, $\left.\ldots, W_{m k 4}\right)$ such that $m=1,2, \ldots, 13$ and $k=1,2, \ldots 224$ and

$W_{m k 1}=\min _{k}\left\{W_{m k 1}\right\}$ 
$W_{m k 2}=\frac{1}{k} \quad \sum_{k=1}^{K} W_{m k 2}$
$W_{m k 3}=\frac{1}{k} \quad \sum_{k=1}^{K} W_{m k 3}$
$W_{m k 4}=\operatorname{maxk}_{k}\left\{W_{m k 4}\right\}$

All the ratings given for each barrier, $C_{j}$, given by all the two hundred twenty-four respondents are clustered together vertically and forming two hundred twenty-four fuzzy sets. For example, the respondents $P_{1}, P_{2}, P_{3}, \ldots, P_{k}$ given the ratings for the $1^{\text {st }}$ barrier $\mathrm{C}_{1}$ as $(0.50 .60 .70 .8),(0.50 .6$ $0.70 .8),(0.40 .50 .50 .6), \ldots,(0.40 .50 .50 .6)$. Similarly, it is done for the rest of the seventeen barriers and the thirteen ISR $A_{m}$. Now, $W_{j k 1}$ is the lowest limit of the aggregate fuzzy weight, the minimum of the lowest numbers for these two hundred twenty-four fuzzy sets, i.e. minimum of $(0.5$, $0.5,0.4, \ldots$, and 0.4$)$ is 0 for the $1^{\text {st }}$ barrier. Similarly, it is calculated for rest barriers and the ISR. Next, $W_{j k 4}$ is the highest limit of aggregate fuzzy weight: the maximum of $0.8,0.8,0.6, \ldots$ and 0.6 , that is 1 . The rest two in-between numbers of the aggregate fuzzy weight are calculated as $W_{j k 2}=$ $W_{112}=1 / 224(0.6+0.6+0.5+\ldots .+0.5)=0.6143$ and $W_{j k 3}=W_{113}=1 / 224(0.8+0.8+0.6+\ldots+$ $0.6)=0.6924$. Similarly aggregated fuzzy weights are also calculated for the requirements of the system. The weight matrix for both barriers and ISR can be expressed as $W_{J}=\left[\begin{array}{llll}W_{1} & W_{2} & \Lambda & W_{j}\end{array}\right]$ and $W_{m}=\left[\begin{array}{llll}W_{1} & W_{2} & \Lambda & W_{m}\end{array}\right]$ respectively. Referring to Table 5, the aggregate fuzzy weight for the barrier

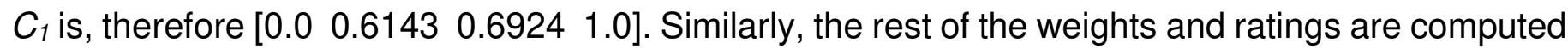
and tabulated for each factor. A similar calculation is done for the aggregate fuzzy weights for the ISR tabulated in Table 6.

Defuzzification is the process of finding an equivalent single crisp value for a fuzzy set. Thus, the aggregated fuzzy weights and rating are to be defuzzified using a suitable defuzzification method. The centre of the area or COA method for Defuzzification is applied here to find the crisp weights and ratings, and the result obtained is plotted in Fig. 7 and Fig 8, respectively. The mathematical explanation for the COA method of Defuzzification is given below:

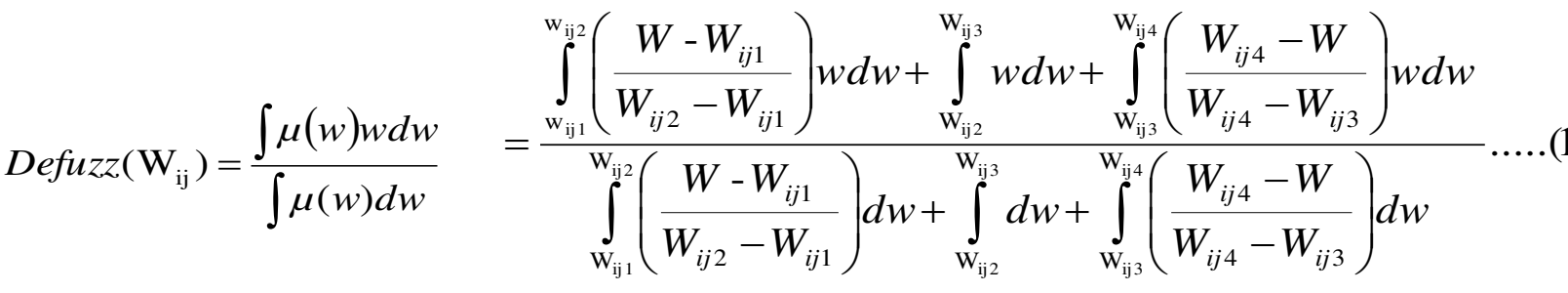

$$
\begin{aligned}
& =\frac{-W_{i j 1} \cdot W_{i j 2}+W_{i j 3} \cdot W_{i j 4}+\frac{1}{3}\left(W_{i j 4}-W_{i j 3}\right)^{2}-\frac{1}{3}\left(W_{i j 2}-W_{i j 1}\right)^{2}}{-W_{i j 1}-W_{i j 2}+W_{i j 3}+W_{i j 4}} \ldots .
\end{aligned}
$$

Similarly, Defuzzification of the fuzzy weights for the ISR is presented in the following relation:

$\operatorname{Defuzz}\left(\mathrm{W}_{\mathrm{im}}\right)=\frac{\int \mu(w) w d w}{\int \mu(w) d w}$

$$
\begin{aligned}
& =\frac{\int_{\mathrm{w}_{\text {im } 1}}^{\mathrm{w}_{\text {im } 2}}\left(\frac{W-W_{i m 1}}{W_{i m 2}-W_{i m 1}}\right) w d w+\int_{\mathrm{W}_{\text {im } 2}}^{\mathrm{W}_{\text {im } 3}} w d w+\int_{\mathrm{W}_{\text {im } 3}}^{\mathrm{W}_{\text {im }}}\left(\frac{W_{i m 4}-W}{W_{i m 4}-W_{i m 3}}\right) w d w}{\int_{\mathrm{W}_{\text {im } 1}}^{\mathrm{W}_{\text {im } 2}}\left(\frac{W-W_{i m 1}}{W_{i m 2}-W_{i m 1}}\right) d w+\int_{\mathrm{W}_{\text {im } 2}}^{\mathrm{W}_{\text {im } 3}} d w+\int_{\mathrm{W}_{\mathrm{im} 3}}^{\mathrm{W}_{\mathrm{im} 4}}\left(\frac{W_{i j 4}-W}{W_{i j 4}-W_{i m 3}}\right) d w} \\
& =\frac{-W_{i m 1} \cdot W_{i m 2}+W_{i m 3} \cdot W_{i m 4}+\frac{1}{3}\left(W_{i m 4}-W_{i m 3}\right)^{2}-\frac{1}{3}\left(W_{i m 2}-W_{i m 1}\right)^{2}}{-W_{i m 1}-W_{i m 2}+W_{i m 3}+W_{i m 4}} \ldots
\end{aligned}
$$


Calculating defuzzification, for example, the aggregated fuzzy rating corresponding to barrier $C 1$ is $\left[\begin{array}{lll}0.0 & 0.6143 & 0.6924 \\ 1.0\end{array}\right]$. Hence, $W_{i j 1}=0.0, W_{i j 2}=0.6143, W_{i j 3}=0.6924$ and $W_{i j 4}=1.0$. Using the expression (2) for defuzzification by COA method, we get,

$$
C_{C 1}=\frac{-(0.0 \cdot 0.6143)+(0.6924 \cdot 1.0)+\frac{1}{3}(1.0-0.6924)^{2}-\frac{1}{3}(0.6143-0.0)^{2}}{-0.0-0.6143+0.6924+1.0}=0.55482
$$

Similarly, the rest of the factors' crisp values are computed and then the results are plotted in the column chart for better understanding, as shown in Fig. 7 and Fig. 8, respectively. Each of the crisp values is arranged in descending order and tabulated in Table 6 and Table 7.

\subsection{Discussion Part 1}

The initial phase of the implementation of Industry 4.0 technologies completely depends on effective solutions to the top dominant factors, as obtained in Table 6 and 7. Thus, out of all the identified barriers and industrial system requirements, our main area of interest is focused on the top three factors as per their rankings in the Multi-Criteria Analysis, which motivates the researchers to focus on these dominant factors and find effective solutions for implementation. The top three dominant barriers that affect Industry 4.0 and its core technologies implementation in the present manufacturing industries, followed by the top three most existing Industrial systems requirements, are discussed below. This analysis helps the decision-makers, researchers and industrialists to focus on these findings.

1. From Table 6, we find criterion $C$ 3, the identified barrier - Employee flexibility required to learn and adapt becomes the most dominant barrier that affects the implementation of 14.0 technologies in the Indian Manufacturing Industries. 14.0 is the latest technology consisting of digitalization of traditional systems, connectivity, data-centric, information and technology, internet of things and data-driven. Before the industrial system upgradation, the current employee should adopt the latest skills and latest technology-driven software and hardware. Thus, the employees need to upgrade their skills, learn data analysis, languages, acquainted with the latest technologies to monitor the new systems, and give their valuable inputs for efficient internet of things solutions. Most of the employees working in a traditional environment find difficulties and hesitant while upgrading themselves to multi-skills. The organization's employer finds it challenging to motivate the experienced employees, especially those who are reluctant to adopt the upgradation due to uncertainty about its future implications, thus creating disruptions and fear of losing their job. So, the debate between the organisation's officials, employees, contractors, and management becomes a major challenge to adapt 14.0 technologies in IMI. This challenge needs to be addressed and must be given the highest priority to develop a proper solution for the adaption of 14.0 technologies.

2. The next dominant barrier comes C 6, Integrating physical systems with cyber systems. As discussed in Section 2 and 3, Cyber-Physical System is an entirely data-driven application that inter-connects the virtual space with physical components of the systems through integrated computing, complex algorithms, smart sensors data, embedded systems, communication, big data, data analytics and storage capabilities with the help of complex networks and internet. Most of the IMI, especially Micro, Small and Medium Enterprise (MSMEs), is still in the post-second industrial revolution. So, the upgradation of conventional systems and investing capitals in these enterprises is a major challenge. Large Enterprises such as Automobile Manufacturer, Original Equipment Manufacturer (OEM), Electronics Goods and Gadgets Manufacturer are likely to adapt 14.0 technology. But still end to end digital integration, real-time data sharing, installation and upgradation of smart sensors, smart actuators, new software and hardware in existing manufacturing systems without halting complete production is also challenging. Major research work needs to be carried out for 
providing a robust standard solution for implementing cyber systems and integrating them into physical systems in manufacturing industries.

3. Then the next dominant barrier, C 10, real-time full data sharing and monitoring among various units, customers, suppliers, logistics and few selected data sharing with other industries. The main point of concern is the huge storage of big data, continuous analysis, and virtual simulation that is digital twin using real-time data for improving efficiency and prediction of defects and machine tool failure. Cybersecurity, data privacy, and continuous monitoring of shared real-time data create a huge challenge to implement 14.0 technologies in existing manufacturing systems. Extensive and empirical research works need to be carried out, especially by the solution-based consultancy currently working on this issue.

4. Table 7, we find that the industrial system requirement A6, Transparency through real-time data monitoring and exchange, Industrial experts believed that it is highly required in the existing manufacturing industries. As in existing Indian manufacturing systems, there is no proper transparency in data collection or any information shared among the systems; hence, human interference increases and causing bottleneck issues, delay, defects in parts and products, etc. So, the data collected from sensors, components, devices of the manufacturing systems, human operators and tool systems need to be interconnected with a communicating medium to provide continuous feedback and monitoring from the real-time data during manufacturing. This helps improve and update the existing production process and send instructions to use the updated data in the next production batch. These multiple data and analysis in real-time assists the manufacturing systems to know the customer, their acquisition and predicting their type of demand and understanding the behaviour towards the products, which is highly essential in existing manufacturing scenario.

5. This is followed by the ISR factor A 5, which is Automatic data exchange and communication. The respondents from various organisations believe that after real-time data transparency through interconnected networks, it's high time, looking at the fluctuating and aggressive market competition, the systems to communicate with each other and make decisions without any human intervention. These systems automatically receive and delivers the right data to the right place at the right time. This saves a generous amount of time and ultimately improves productivity. As the systems are interconnected through networks, these can make their own decision and share the data to the right place at the right time without any human interference.

6. Then the $3^{\text {rd }}$ most essential system requirement is the factor $A 10$, that is, Improving productivity. In general, every industrial expert wants their existing productivity to be improved either through traditional optimisation methods, industrial management and other techniques or by implementing advanced technology, re-tooling to latest equipment and hardware and upgrading and reforming their existing systems. With the application of 14.0 technologies such as advanced continuous monitoring process, machine to machine communication, real-time data sharing, data analytics and digital twin, the chances of system breakdown, prediction for maintenance of tools and equipment, predictive analysis of the failure of the components, proper resource management leads to improving productivity. It is highly required in current manufacturing industries.

\subsection{Co-Relation Analysis}

The inter-item co-relation analysis is performed for barriers' $C$ ' and systems requirements ' $A$ ' separately to investigate the linear relationship between the variables and observe how closely each variable is related to the other. Then, the inter-item individual co-relation analysis was performed between barriers and systems requirements to determine how closely the ISR is related to the barriers and vice versa. All the analysis was computed, and the results were tabulated in Table 8, 9 and 10. The primary analysis is focused on the correlation between barriers and systems requirements of Industry 4.0 and drawing out conclusions from it. The correlation coefficient ranges 
from -1.0 , a perfect negative correlation, to +1 , a perfect positive correlation. The more the value gets closure to -1.0 or 1.0 , the stronger the relationship between the inter-item variables; if the correlation coefficient is 0 , then there is a weaker correlation (Meyers et al., 2013). A higher degree of co-relation is when its coefficient ranges above 0.75 , moderate when it ranges between 0.25 to 0.50 , and the absence of co-relation ranges 0 to 0.25 in both positive and negative coefficient values (H., Robert, 2015).

\subsection{Discussion Part 2}

As each of the ISR and Barriers variables are obtained and tabulated, it is quite lengthy for explaining every coefficient of both barriers and ISR. So, as per the consideration, the following co-efficient has been chosen randomly for a brief explanation. With this explanation, it can be related to every single variable. The following are the key observation and the discussion that has been pointed out from the analysis.

1. Table 8 and 9 represents the inter-item correlation of each variable. From both the table, it is observed that all the coefficients obtained are positive; that is, all the variables are of barriers, and ISR are positively correlated. For example, in Table 8, the coefficient of correlation of the industrial system requirement factor A6 (Transparency through real-time data monitoring and exchange) and the factor A10 (Improving productivity) is 0.6265 . That is, the variables are positively co-related. If the value of the variable $A 6$ tends to increase, the value of variable A10 would also increase. In a practical case, if the system were interconnected from where the real-time data could be monitored and exchanged with data transparency, it affects by increasing the value of the variable $A 10$; that is, it would increase productivity through continuous monitoring real-time feedback. In this way, every co-efficient of every variable are related. The more the value of the coefficient, the more the variables are related to each other. From the obtained table, the rest of the variables and their relation are concluded, which can be used by different practitioners and solution providers to implement the 14.0 technology in existing manufacturing systems. Similarly, in Table 9, all the variables are positively correlated with each other, some have very less coefficient, and some have very high coefficient.

2. In Table 10, the positive coefficient values are positive co-relations. If the value of one variable increases, then ultimately, the second corresponding variable's value is also increased and vice versa. For example, considering $4^{\text {th }}$ column that is Factor A4 (Improving inter-connectivity among all the systems) and $3^{\text {rd }}$ row that is barrier C3 (Employee flexibility required to learn and adapt) are positively co-related to a co-efficient of 0.195 . In this, if the importance of improving inter-connectivity among all the manufacturing (which the respondent feels is an essential factor that should be present in the current organisation) increases then, it also affects the increasing importance of employee flexibility required to learn and adapt to the new technologies, which acts as a barrier to implement Industry 4.0. In this way, positive correlations are determined and concluded to learn how systems requirements are affected by implementing barriers and vice versa. As there is a prior requirement to find the positive co-relations, the top seven coefficients are selected and highlighted in italics, as shown in Table 13. These are given the highest importance and are considered a referring tool for the practitioners, solution providers, researchers and industrialists to implement Industry 4.0 technologies in their organisation.

3. All the negatively correlated coefficients are marked with an underline, as shown in Table 10. This negative correlation determines that if one variable's values increase, the corresponding variable's value decreases. It generates the type of relations between the factors and barriers which can be given the least importance by the researchers, practitioners, industrialists for analysis and implementation. For example, the implementing factor A2 increases flexibility in scalability and variety in manufacturing systems. The respondents believe it is vital in present 
manufacturing systems, and the barrier $\mathrm{C} 1$ that is the Lack of understanding and knowledge of Industry 4.0 technologies, are negatively related. If the importance of the ISR increases, then simultaneously, this barrier's value decreases and vice versa.

\section{CONCLUSION}

The present research work identifies the most weighted barriers that affect Industry 4.0 implementation and the current ISR necessary for the existing manufacturing systems. This paper points out the relevant areas and narrows them down by minimising the gap between existing ISR and the barriers affecting implementation and prioritising the importance of the research to implement 14.0 technologies. At first, the necessity analysis such as the KMO, Bartlett's test of Sphericity and Descriptive statistics analysis was conducted to check the legitimateness of the identified barriers and ISR data set. It provides a guideline to conduct factor analysis, which could be considered in future works. In the next, Multi-Criteria analysis conducted in the fuzzy environment aids the decision-makers and industrialists identify and address this highest weightage ISR and barriers. That is arranged in decreasing order to give weightage for future research during implementation. The top three dominant barriers that affect Industry 4.0 and its core technologies implementation in the present manufacturing industries, followed by the top three most existing Industrial systems requirements was obtained. It also provides a roadmap to the researchers, practitioners, solution providers for solving MCDM problems to implement 14.0 technologies with weights of the identified ISR and the barriers obtained in this paper. Lastly, the inter-item co-relation between the ISR and the implementing barriers of Industry 4.0 was analysed, which determines the relationship shared by the most dominant positive correlation that is, ISR, The Increasing system responsiveness and the barrier, Quick reconfiguration of manufacturing, assembly systems, inspection and material handling systems. This correlation can be used as a referring tool and a gateway for minimising the gap between ISR and barriers.

In the future, based on these analyses and findings, the authors seek to work on an architectural framework model that can be designed and developed in the context of the implementation barrier and ISR factors for adapting Industry 4.0 in IMI. It can be used as a tool for evaluation in numerous case studies.

\section{DECLARATION}

We wish to submit an original research article entitled "Analysis and Evaluation of Indian Industrial System Requirements and Barriers Affect During Implementation of Industry 4.0 Technologies" for consideration by The International Journal of Advanced Manufacturing Technology. We confirm that this work is original and has not been published elsewhere, nor is it currently considered for publication elsewhere. We have no conflicts of interest to disclose.

\section{a. Funding: Not Applicable \\ b. Conflicts of interest/Competing interests:}

The authors whose names are listed in this manuscript certify that they have NO conflict of interest also NO affiliations with or involvement in any organization or entity with any financial interest (such as honoraria; educational grants; participation in speakers' bureaus; membership, employment, consultancies, stock ownership, or other equity interest; and expert testimony or patent-licensing arrangements), or non-financial interest (such as personal or professional relationships, affiliations, knowledge or beliefs) in the subject matter or materials discussed in this manuscript.

c. Availability of data and material: The data set collected from a questionnaire-based survey as mentioned in section 5.3 and 5.4, respectively.

d. Code availability: Not applicable

e. Ethics approval: Not applicable

f. Consent to participate Not applicable 
g. Consent for publication: Not applicable

h. Authors' contributions: Both the authors mentioned in this manuscript contributed equally to selecting research tools, performing analysis, writing and evaluating this research work.

\section{REFERENCES}

1. Agarwal, R. (2018). Samarth Udyog Digitization of Markets and Industry in India. Retrieved from https://www.samarthudyog-i40.in/

2. Calia, E., \& D'Aprile, D. (2020). Industry4.0. In Analytics for the Sharing Economy: Mathematics, Engineering and Business Perspectives, 309-333. https://doi.org/10.1007/978-3-030-35032-1_18

3. Cheng, G., Liu, L., Qiang, X., \& Liu, Y. (2016). Industry 4.0 Development and Application of Intelligent Manufacturing. International Conference on Information System and Artificial Intelligence (ISAI), Hong Kong, 2016, 407-410. https://doi.org/10.1109/ISAI.2016.0092

4. Cohen, Y., Faccio, M., Pilati, F., \& Yao, X. (2019). Design and management of digital manufacturing and assembly systems in the Industry 4.0 era. The International Journal of Advanced Manufacturing Technology, 105(9), 3565-3577. https://doi.org/10.1007/s00170019-04595-0

5. Dean, M., \& Spoehr, J. (2018). The fourth industrial revolution and the future of manufacturing work in Australia: challenges and opportunities. Labour \& Industry: A Journal of the Social and Economic Relations of Work, 28(3), 166-181. https://doi.org/10.1080/10301763.2018.1502644

6. Fatorachian, H., \& Kazemi, H. (2018). A critical investigation of Industry 4.0 in manufacturing: theoretical operationalisation framework. Production Planning and Control, 29(8), 633-644. https://doi.org/10.1080/09537287.2018.1424960

7. Fettermann, D. C., Cavalcante, C. G. S., Almeida, T. D. De, \& Tortorella, G. L. (2018). How does Industry 4.0 contribute to operations management? Journal of Industrial and Production Engineering, 35(4), 255-268. https://doi.org/10.1080/21681015.2018.1462863

8. Field, A. (2017). Discovering Statistics Using IBM SPSS Statistics. Retrieved from https://books.google.co.in/books?id=QMI-DwAAQBAJ

9. Foundation, I. B. E. (2019). Annual Manufacturing Report. Retrieved from www.ibef.org

10. Frank, A. G., Dalenogare, L. S., Ayala, N. F., Stock, T., Obenaus, M., Kunz, S., Downey, J. (2018). Industry 4.0 technologies: Implementation patterns in manufacturing companies. International Journal of Production Research, 6(1), 1-10. https://doi.org/10.1016/j.jii.2017.04.005

11. George, D., \& Mallery, P. (2018). IBM SPSS Statistics 25 Step by Step. In IBM SPSS Statistics 25 Step by Step. https://doi.org/10.4324/9781351033909

12. Ghobakhloo, M. (2018). The future of manufacturing industry: a strategic roadmap toward Industry 4.0. Journal of Manufacturing Technology Management, 29(6), 910-936, https://doi.org/10.1108/JMTM-02-2018-0057

13. Gilchrist, A., Gilchrist Bangken, A., \& Thailand, N. (2016). THE INDUSTRIAL INTERNET OF THINGS Industry 4.0: The Industrial Internet of Things. In Library of Congress Control Number. https://doi.org/10.1007/978-1-4842-2047-4

14. Hu, S. J., Zhu, X., Wang, H., \& Koren, Y. (2008). CIRP Annals - Manufacturing Technology Product variety and manufacturing complexity in assembly systems and supply chains. 57, 45-48. https://doi.org/10.1016/j.cirp.2008.03.138

15. Iglesias-Urkia, M., Orive, A., Barcelo, M., Moran, A., Bilbao, J., \& Urbieta, A. (2017). Towards a lightweight protocol for Industry 4.0: An implementation based benchmark. Proceedings of the 2017 IEEE International Workshop of Electronics, Control, Measurement, Signals and $\begin{array}{llll}\text { Their Application to Mechatronics, } & \text { ECMSM }\end{array}$ https://doi.org/10.1109/ECMSM.2017.7945894

16. India's Readiness for Industry 4.0 - A Focus on Automotive Sector. (2017). In Grant Thornton. Retrieved from http://www.grantthornton.in/insights/articles/indias-readiness-forindustry-4.0--a-focus-on-automotive-sector/ 
17. India Brand Equity Foundation. (2016). The Best of India in Engineering. 9. Retrieved from https://www.ibef.org/the-best-of-india-in-engineering-2017.pdf

18. Industry 4.0 India Inc. gearing up for change. (2018) AIMA-KPMG Industry 4.0 Report, Retrieved from http://resources.aima.in/presentations/AIMA-KPMG-industry-4-0-report.pdf .

19. Iyer, A. (2018). Moving from Industry 2.0 to Industry 4.0: A case study from India on leapfrogging in smart manufacturing. Procedia Manufacturing, 21, 663-670. https://doi.org/10.1016/j.promfg.2018.02.169

20. Kamble, S., Gunasekaran, A., \& Dhone, N. C. (2019). Industry 4.0 and lean manufacturing practices for sustainable organisational performance in Indian manufacturing companies. International Journal of Production Research, 58(5), 1-19. https://doi.org/10.1080/00207543.2019.1630772

21. Kamble, S. S., Gunasekaran, A., \& Gawankar, S. A. (2018). Sustainable Industry 4.0 framework: A systematic literature review identifying the current trends and future perspectives. Process Safety and Environmental Protection, 117, 408-425. https://doi.org/10.1016/j.psep.2018.05.009

22. Kamble, S. S., Gunasekaran, A., \& Sharma, R. (2018). Analysis of the driving and dependence power of barriers to adopt industry 4.0 in Indian manufacturing industry. Computers in Industry, 101(June), 107-119. https://doi.org/10.1016/j.compind.2018.06.004

23. Lasi, H., \& Kemper, H.-G. (2019). Industry 4.0. Business \& Information Systems Engineering, 56(4), 239-242. https://doi.org/10.1007/978-981-13-3384-2_13

24. Laudante, E. (2017). Industry 4.0, Innovation and Design. A new approach for ergonomic analysis in manufacturing system. The Design Journal, 20(1), 2724-2734. https://doi.org/10.1080/14606925.2017.1352784

25. Li, L. (2018). China's manufacturing locus in 2025: With a comparison of "Made-in-China 2025" and "Industry 4.0." Technological Forecasting and Social Change, 135(May 2017), 6674. https://doi.org/10.1016/j.techfore.2017.05.028

26. Li, L., Chen, C. L. H., Lasi, H., Kemper, H.-G., Pikkarainen, M., Pekkarinen, S., ... Anderl, R. (2018). Industry 4.0 in management studies: A systematic literature review. International Journal of Production Research, 6(1), 1-10. https://doi.org/10.1016/j.jii.2017.04.005

27. Liao, Y., Deschamps, F., Loures, E. de F. R., \& Ramos, L. F. P. (2017). Past, present and future of Industry 4.0 - a systematic literature review and research agenda proposal. International Journal of Production Research, 55(12), 3609-3629. https://doi.org/10.1080/00207543.2017.1308576

28. Liu, P., \& Jin, F. (2012). A multi-attribute group decision-making method based on weighted geometric aggregation operators of interval-valued trapezoidal fuzzy numbers. Applied Mathematical Modelling, 36(6), 2498-2509. https://doi.org/10.1016/j.apm.2011.09.006

29. Lotter B., Wiendahl HP. (2009) Changeable and Reconfigurable Assembly Systems. In: EIMaraghy $H$. (eds) Changeable and Reconfigurable Manufacturing Systems. Springer Series in Advanced Manufacturing. Springer, London, 127-142. https://doi.org/10.1007/9781-84882-067-8_7

30. Lu, Y. (2017). Industry 4.0: A survey on technologies, applications and open research issues. Journal of Industrial Information Integration, 6, 1-10. https://doi.org/10.1016/j.jii.2017.04.005

31. Luthra, S., \& Mangla, S. K. (2018). Evaluating challenges to Industry 4.0 initiatives for supply chain sustainability in emerging economies. Process Safety and Environmental Protection, 117, 168-179. https://doi.org/10.1016/j.psep.2018.04.018

32. Malhotra, M. K., \& Grover, V. (1998). An assessment of survey research in POM: From constructs to theory. Journal of Operations Management, 16(4), 407-425. https://doi.org/10.1016/s0272-6963(98)00021-7

33. Matt D.T., Rauch E. (2020) SME 4.0: The Role of Small- and Medium-Sized Enterprises in the Digital Transformation. In: Matt D., Modrák V., Zsifkovits H. (eds) Industry 4.0 for SMEs. Palgrave Macmillan, Cham, 3-36. https://doi.org/10.1007/978-3-030-25425-4_1

34. Meyers, L. S., Gamst, G. C., \& Guarino, A. J. (2013). Performing Data Analysis Using IBM SPSS. Retrieved from https://books.google.co.in/books?id=SQgeAAAAQBAJ

35. Ministry of Statistics and Program Implementation.

(2019). https://doi.org/http://mospi.nic.in/sites/default/files/iip/Indices\%20IIP2011- 
12\%20Monthly_annual18082020.xIsx

36. Moeuf, A., Lamouri, S., Pellerin, R., Tamayo, G.S., Tobon V.E., \& Eburdy, R. (2019). Identification of critical success factors, risks and opportunities of Industry 4.0 in SMEs. International Journal of Production Research, 58(5), 1384-1400. https://doi.org/10.1080/00207543.2019.1636323

37. Moktadir, M. A., Ali, S. M., Kusi-Sarpong, S., \& Shaikh, M. A. A. (2018). Assessing challenges for implementing Industry 4.0: Implications for process safety and environmental protection. Process Safety and Environmental Protection, 117, 730-741. https://doi.org/10.1016/j.psep.2018.04.020

38. Mueller, E., Chen, X. L., \& Riedel, R. (2017). Challenges and Requirements for the Application of Industry 4.0: A Special Insight with the Usage of Cyber-Physical System. Chinese Journal of Mechanical Engineering (English Edition), 30(5), 1050-1057. https://doi.org/10.1007/s10033-017-0164-7

39. Musani, S., \& Jemain, A. A. (2017). A Fuzzy MCDM Approach for Evaluating School Performance Based on Linguistic Information. AIP Conference Proceedings 1571, 10061012. https://doi.org/10.1063/1.4858785

40. Oesterreich, T. D., \& Teuteberg, F. (2016). Understanding the implications of digitisation and automation in the context of Industry 4.0: A triangulation approach and elements of a research agenda for the construction industry. Computers in Industry, 83, 121-139. https://doi.org/10.1016/j.compind.2016.09.006

41. Pattanaik, L. N., \& Jena, A. (2019). Tri-objective optimisation of mixed model reconfigurable assembly system for modular products. International Journal of Computer Integrated Manufacturing, 32(1), 72-82. https://doi.org/10.1080/0951192X.2018.1550673

42. Pereira, A. C., \& Romero, F. (2017). A review of the meanings and the implications of the Industry $4.0 \quad$ concept. Procedia Manufacturing, 13, 1206-1214. https://doi.org/10.1016/j.promfg.2017.09.032

43.Petit, J.-P., Brosset, P., \& Bagnon, P. (2019). Smart factories@ scale. Capgemini, 2019. Retrieved from https://www.capgemini.com/wp-content/uploads/2019/11/Report---SmartFactories.pdf

44. Pico-Valencia, P., \& Holgado-Terriza, J. A. (2018). Agentification of the Internet of Things: A systematic literature review. International Journal of Distributed Sensor Networks, 14(10). https://doi.org/10.1177/1550147718805945

45. Ho R., Popović, B. V. (2015). Handbook of univariate and multivariate data analysis with IBM SPSS, second edition. Journal of Applied Statistics, 42(10), 2291-2291. https://doi.org/10.1080/02664763.2015.1015811

46. Roblek, V., Meško, M., \& Krapež, A. (2016). A Complex View of Industry 4.0. SAGE Open, 6(2), 1-11. https://doi.org/10.1177/2158244016653987

47. Rojko, A. (2017). Industry 4.0 concept: Background and overview. International Journal of Interactive Mobile Technologies, 11(5), 77-90. https://doi.org/10.3991/ijim.v11i 5.7072

48. Salam, M. A. (2019). Analysing manufacturing strategies and Industry 4.0 supplier performance relationships from a resource-based perspective. Benchmarking: An International Journal. https://doi.org/10.1108/BIJ-12-2018-0428

49.Sanders, A., Elangeswaran, C., \& Wulfsberg, J. (2016). Industry 4.0 implies lean manufacturing: Research activities in industry 4.0 function as enablers for lean manufacturing. Journal of Industrial Engineering and Management, 9(3), 811. https://doi.org/10.3926/jiem.1940

50. Santos, C., Mehrsai, A., Barros, A. C., Araújo, M., \& Ares, E. (2017). Towards Industry 4.0: an overview of European strategic roadmaps. Procedia Manufacturing, 13, 972-979. https://doi.org/10.1016/j.promfg.2017.09.093

51. Schmidt, R., Möhring, M., Härting, R. C., Reichstein, C., Neumaier, P., \& Jozinović, P. (2015). Industry 4.0 - Potentials for creating smart products: Empirical research results. Lecture Notes in Business Information Processing, 208, 16-27. https://doi.org/10.1007/978-3-31919027-3_2

52. Sevinç, A., Gür, Ş., \& Eren, T. (2018). Analysis of the Difficulties of SMEs in Industry 4.0 Applications by Analytical Hierarchy Process and Analytical Network Process. Processes, 
6(12), 264. https://doi.org/10.3390/pr6120264

53. Shrouf, F., Ordieres, J., \& Miragliotta, G. (2014). Smart factories in Industry 4.0: A review of the concept and of energy management approached in production based on the Internet of Things paradigm. IEEE International Conference on Industrial Engineering and Engineering Management, 2015 January, 697-701. https://doi.org/10.1109/IEEM.2014.7058728

54. Singla, A., Ahuja, I. S., \& Sethi, A. S. (2019). An examination of effectiveness of technology push strategies for achieving sustainable development in manufacturing industries. Journal of Science and Technology Policy Management, 10(1), 73-101. https://doi.org/10.1108/JSTPM-10-2017-0048

55. Sony, M., \& Naik, S. S. (2019). Ten Lessons for Managers while Implementing Industry 4.0. IEEE Engineering Management Review, 47(2), 45-52. https://doi.org/10.1109/EMR.2019.2913930

56. Telukdarie, A., Buhulaiga, E., Bag, S., Gupta, S., \& Luo, Z. (2018). Industry 4.0 implementation for multinationals. Process Safety and Environmental Protection, 118. https://doi.org/10.1016/j.psep.2018.06.030

57. Tortorella, G. L., \& Fettermann, D. (2018). Implementation of industry 4.0 and lean production in brazilian manufacturing companies. International Journal of Production Research, 56(8), 2975-2987. https://doi.org/10.1080/00207543.2017.1391420

58. Ustundag, A., \& Cevikcan, E. (2018). Industry 4.0: Managing The Digital Transformation. https://doi.org/10.1007/978-3-319-57870-5

59. Venkatesh, Gumaste Vinodh. 2018. "Project Samarth Udyog." Retrieved from https://www.samarthudyog-i40.in/project.

60. Wang, S., Wan, J., Li, D., \& Zhang, C. (2016). Implementing Smart Factory of Industrie 4.0: An Outlook. International Journal of Distributed Sensor Networks,12(1), 1-10. https://doi.org/10.1155/2016/3159805

61. Wang, T. (2006). Multi-Criteria Decision Analysis by Using Fuzzy VIKOR.

62. Wang, T.C., Liang, L., \& Ho, C. (2006). Multi-Criteria decision analysis by using fuzzy VIKOR. Proceedings - ICSSSM'06: 2006 International Conference on Service Systems and Service Management, 2, 901 - 906. 10.1109/ICSSSM.2006.320751.

63. Wang, W., \& Koren, Y. (2012). Scalability planning for reconfigurable manufacturing systems. Journal of Manufacturing Systems, 31(2), 83-91. https://doi.org/10.1016/j.jmsy.2011.11.001

64. Xu, L. D, Xu, E. L., \& Li, L. (2018). Industry 4.0: State of the art and future trends. International Journal of Production Research, 56(8), 2941-2962. https://doi.org/10.1080/00207543.2018.1444806

65.Xu, J., Huang, E., Hsieh, L., Lee, L. H., Jia, Q. S., \& Chen, C. H. (2016). Simulation optimisation in the era of Industrial 4.0 and the Industrial Internet. Journal of Simulation, 10(4), 310-320. https://doi.org/10.1057/s41273-016-0037-6

66. Yin, Y., Stecke, K. E., \& Li, D. (2018). The evolution of production systems from Industry 2.0 through Industry 4.0. International Journal of Production Research, 56(1-2), 848-861. https://doi.org/10.1080/00207543.2017.1403664

67. Zhang, C., Ma C., Xu J. (2005). A New Fuzzy MCDM Method Based on Trapezoidal Fuzzy AHP and Hierarchical Fuzzy Integral. In: Wang L., Jin Y. (eds) Fuzzy Systems and Knowledge Discovery, Lecture Notes in Computer Science, 3614, 466-474 Springer, Berlin, Heidelberg. https://doi.org/10.1007/11540007_57

68. Zhang, A., Pikas, B., \& Lee, T. (2016). The Transformation and Upgrading of the Chinese Manufacturing Industry: Based on "German Industry 4.0." The Journal of Applied Business and Economics, 18(5), 97-105. Retrieved from https://search.proquest.com/docview/1855298156?accountid=38738

69.Zhang, Xin, Jin, F., \& Liu, P. (2013). A grey relational projection method for multi-attribute decision making based on intuitionistic trapezoidal fuzzy number. Applied Mathematical Modelling, 37(5), 3467-3477. https://doi.org/10.1016/j.apm.2012.08.012

70. Zhou, K., Liu, T., \& Zhou, L. (2016). Industry 4.0: Towards future industrial opportunities and challenges. 2015 12th International Conference on Fuzzy Systems and Knowledge Discovery, 2147-2152. https://doi.org/10.1109/FSKD.2015.7382284 

Figures

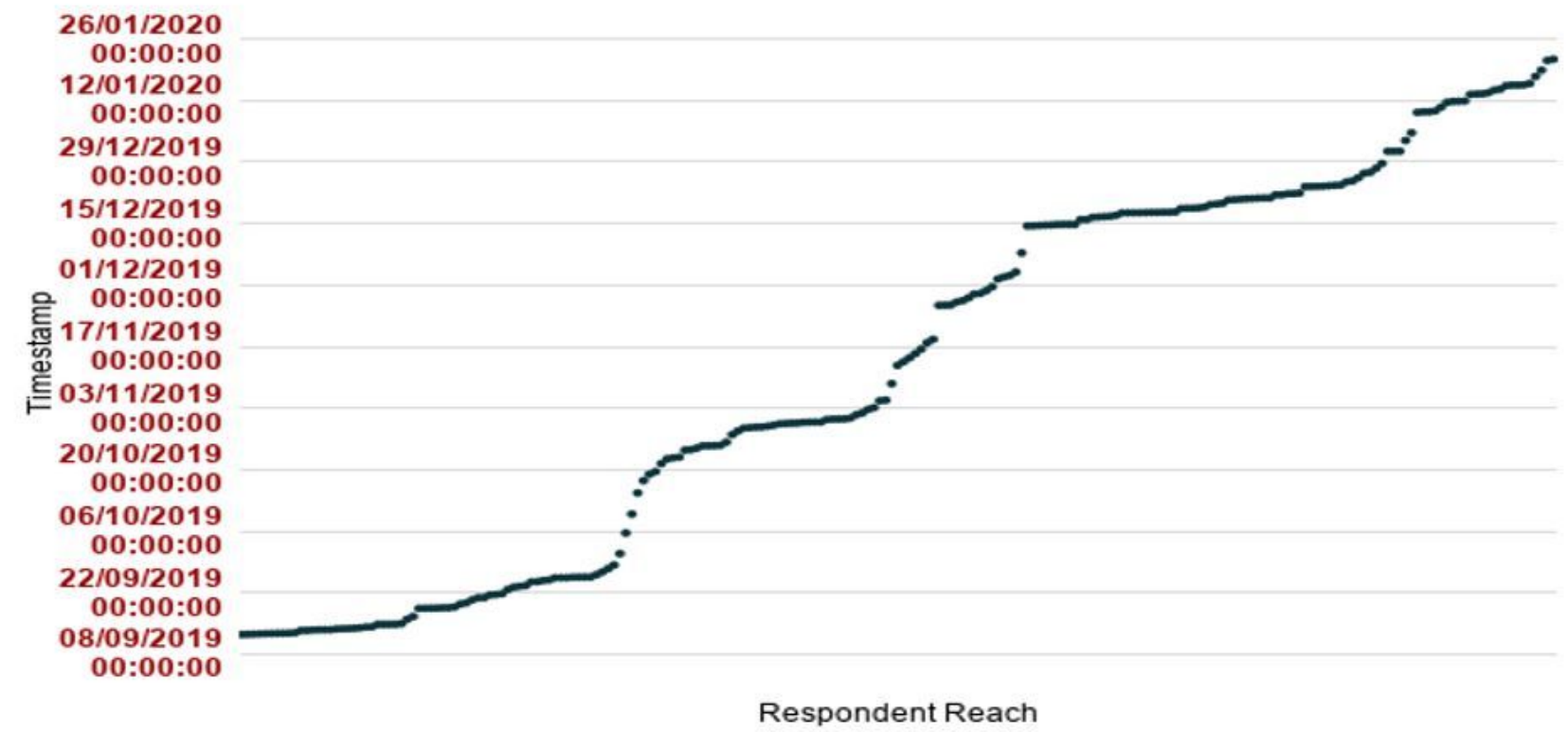

\section{Figure 1}

Timeline of the survey response received

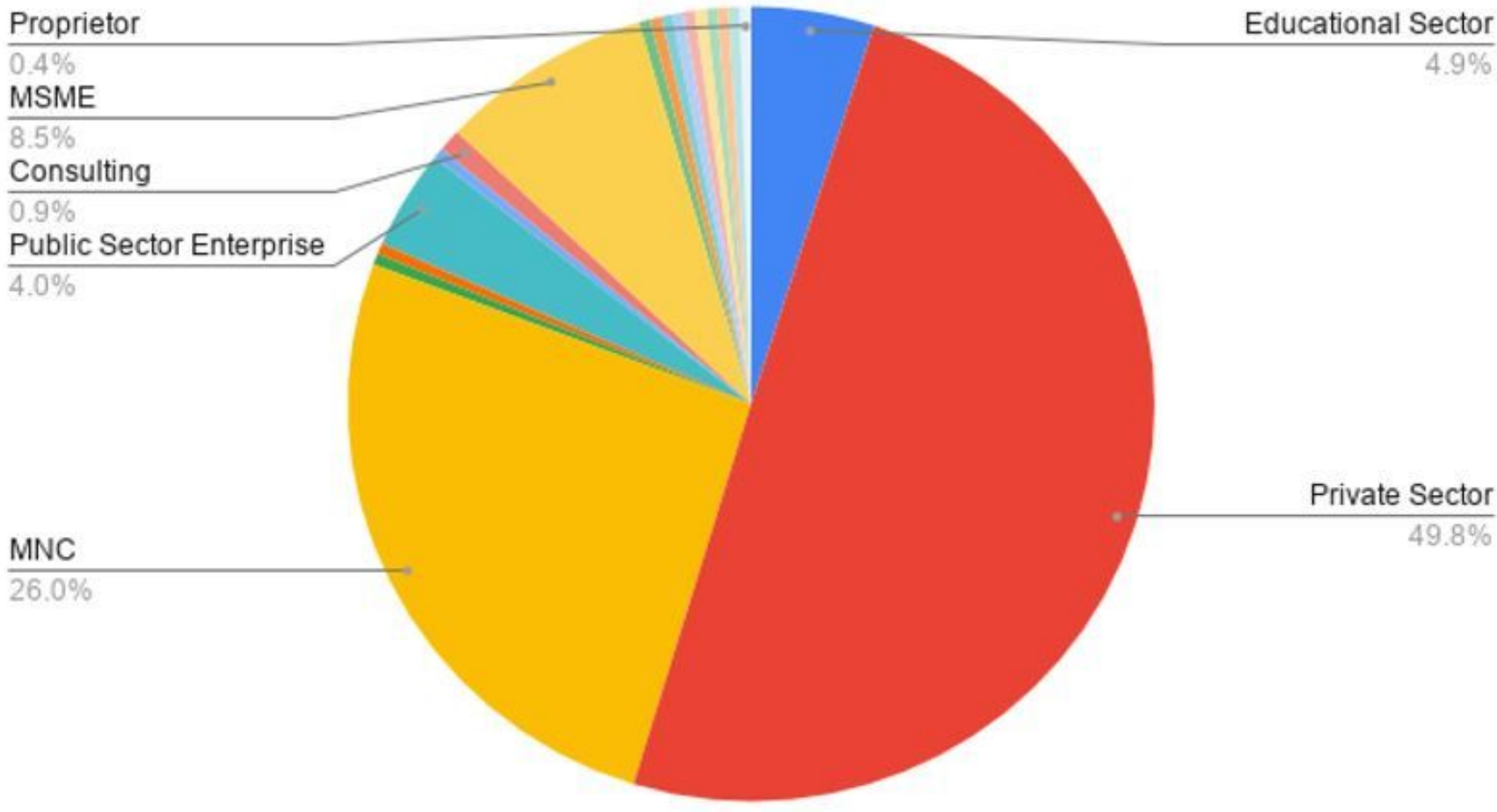


Figure 2

Types of organisation

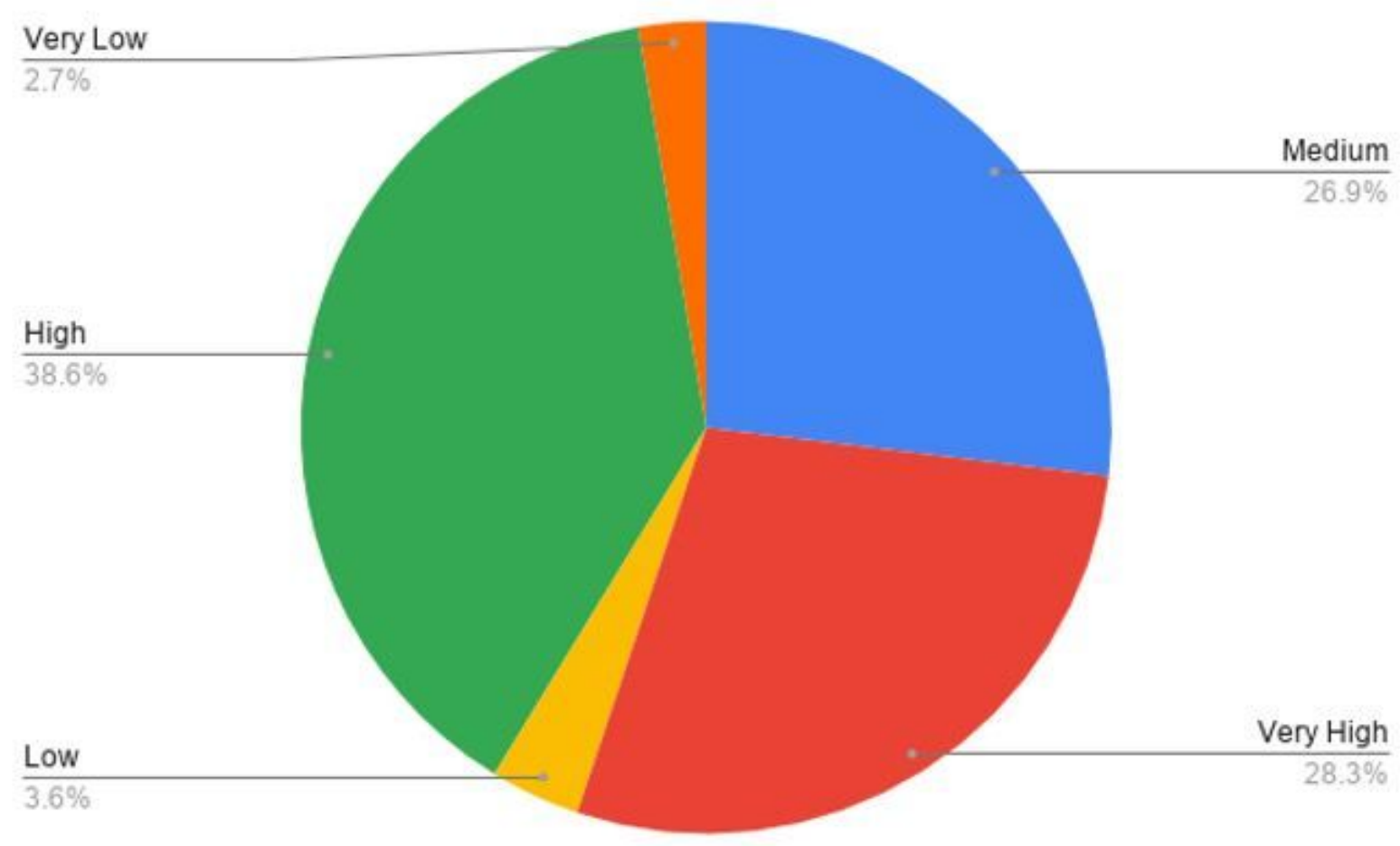

Figure 3

Respondents aware of Industry 4.0 


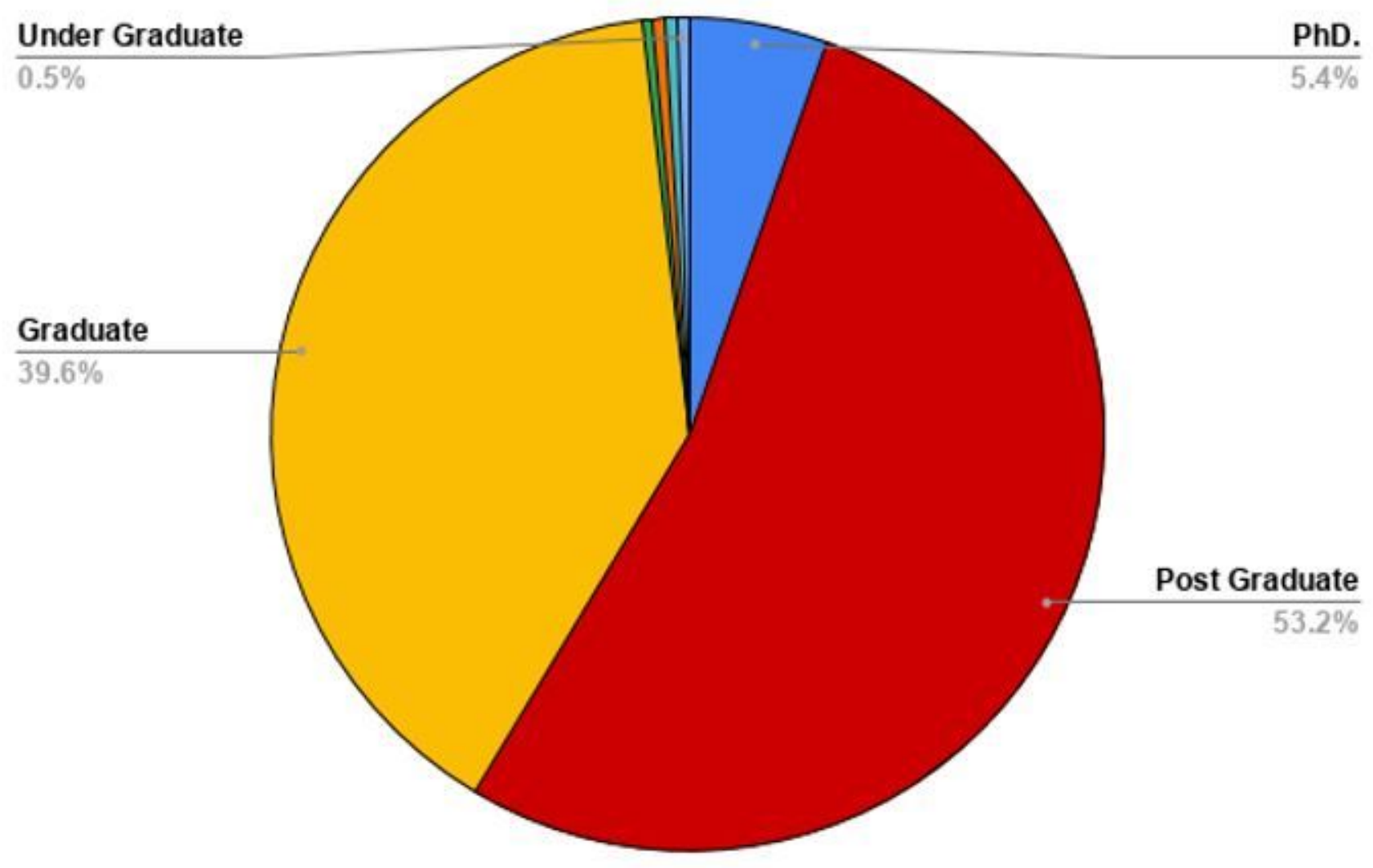

Figure 4

Respondent's Educational Background

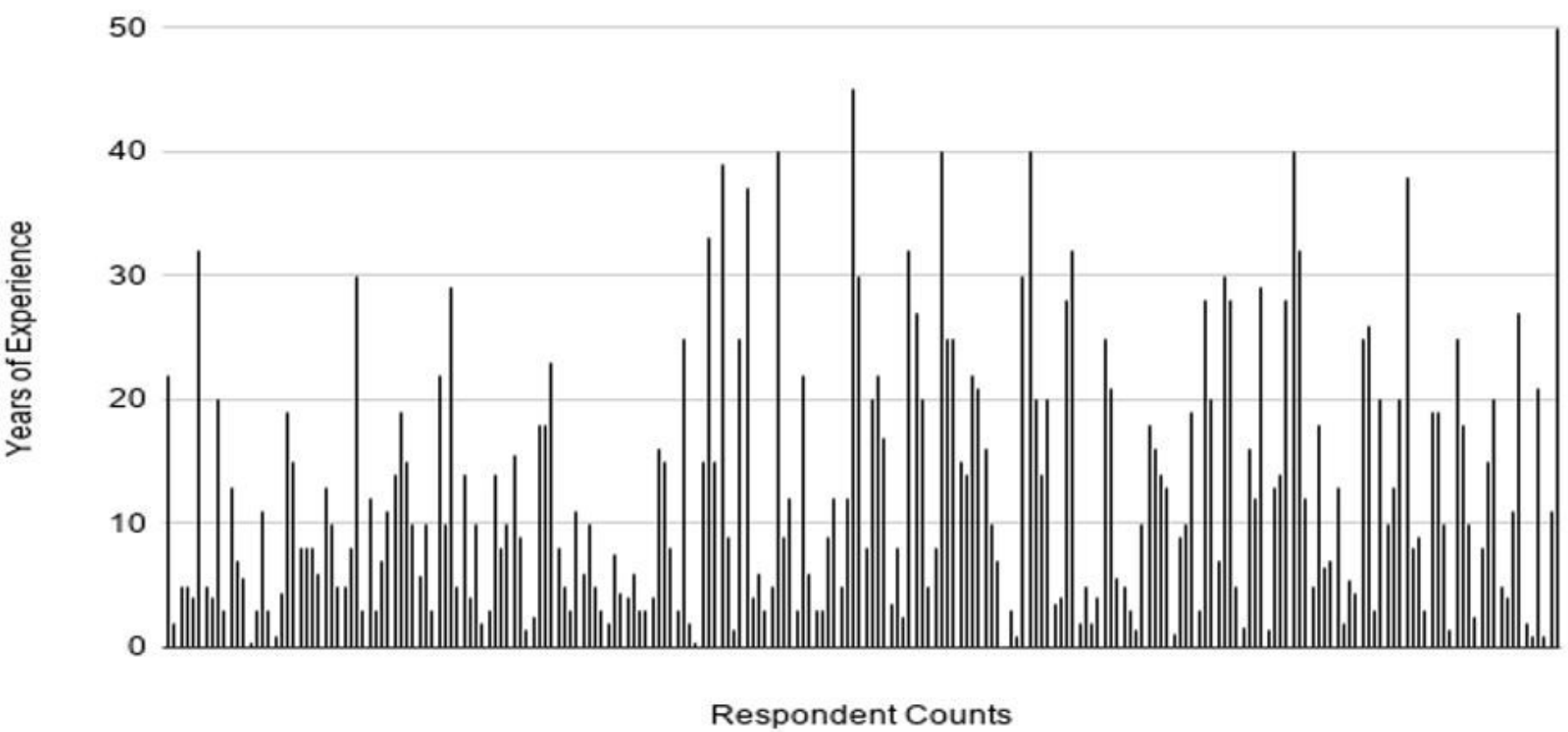


Figure 5

Years of Experience

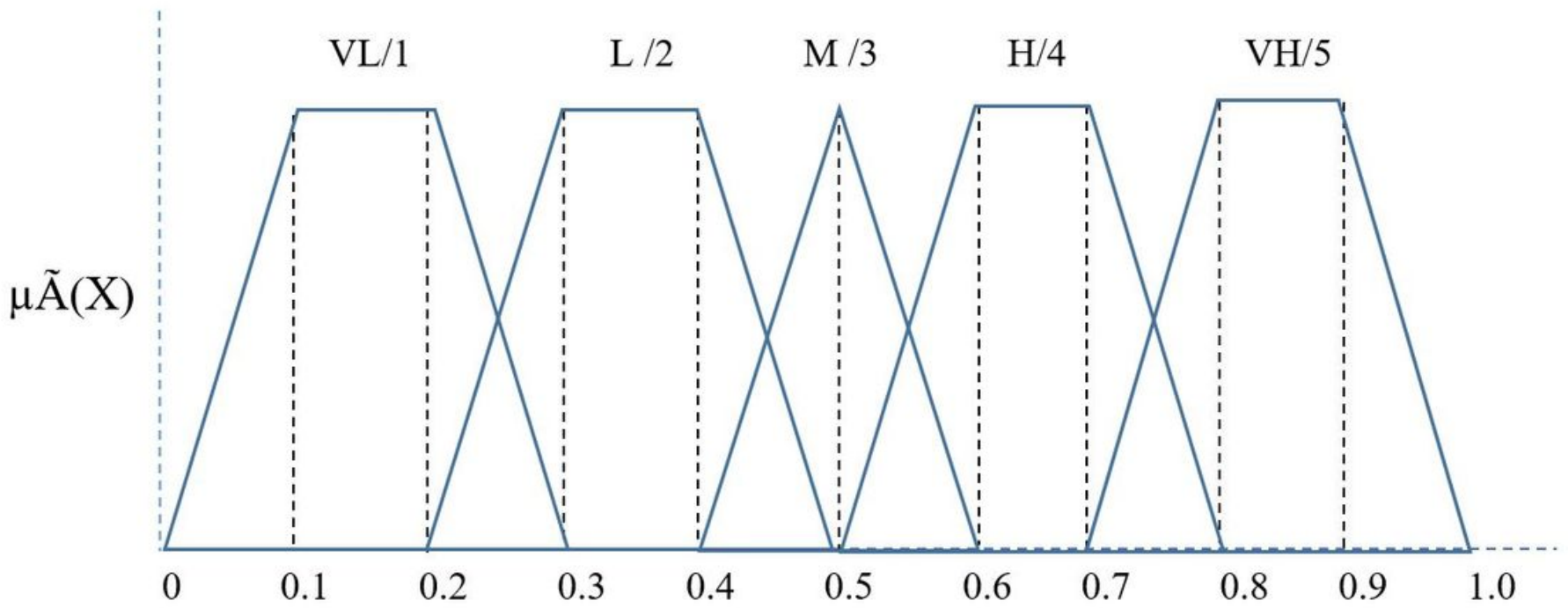

Figure 6

Linguistic variables

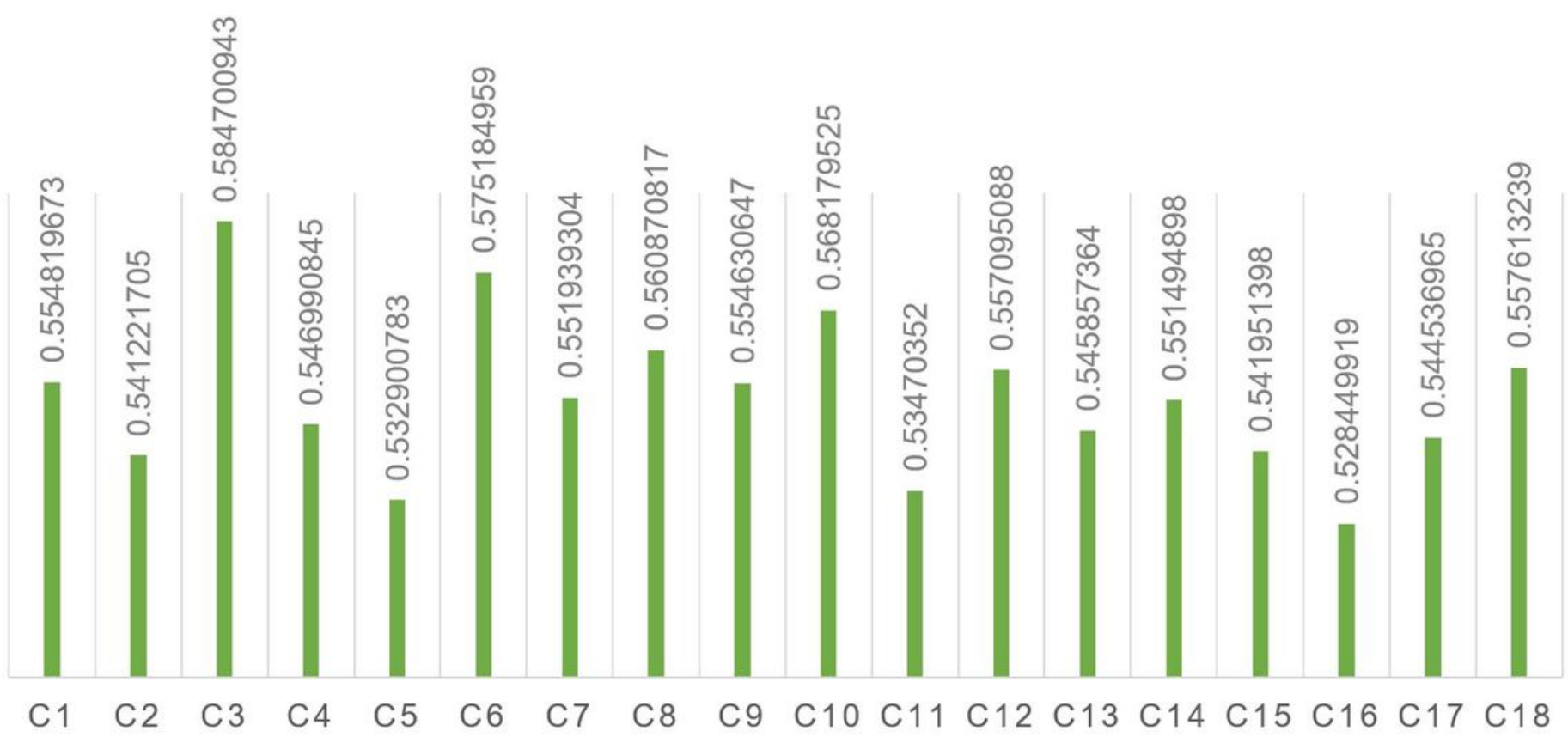

Figure 7

Crisp Values of Barriers 


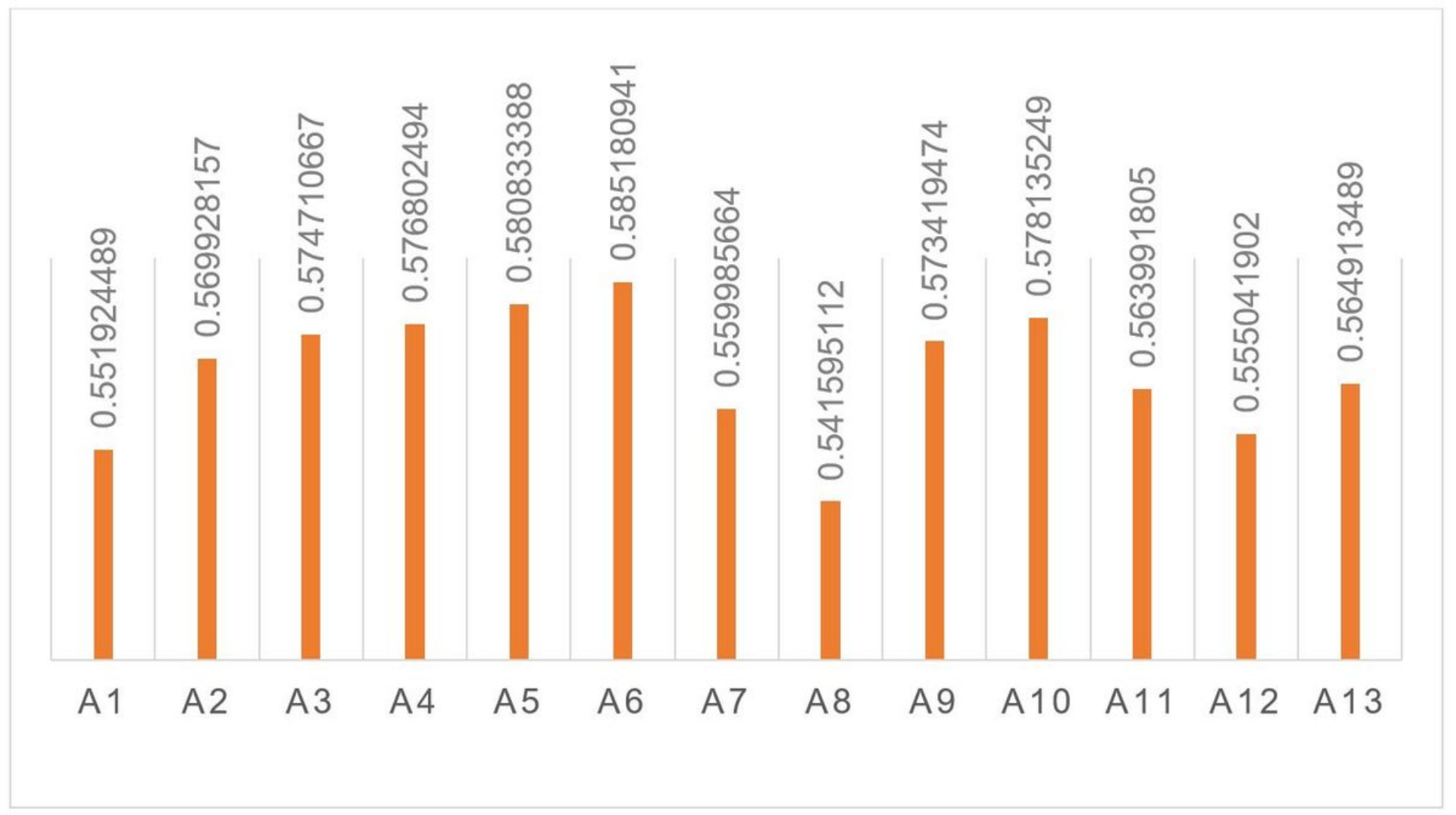

Figure 8

Crisp Values of Industrial System Requirements 


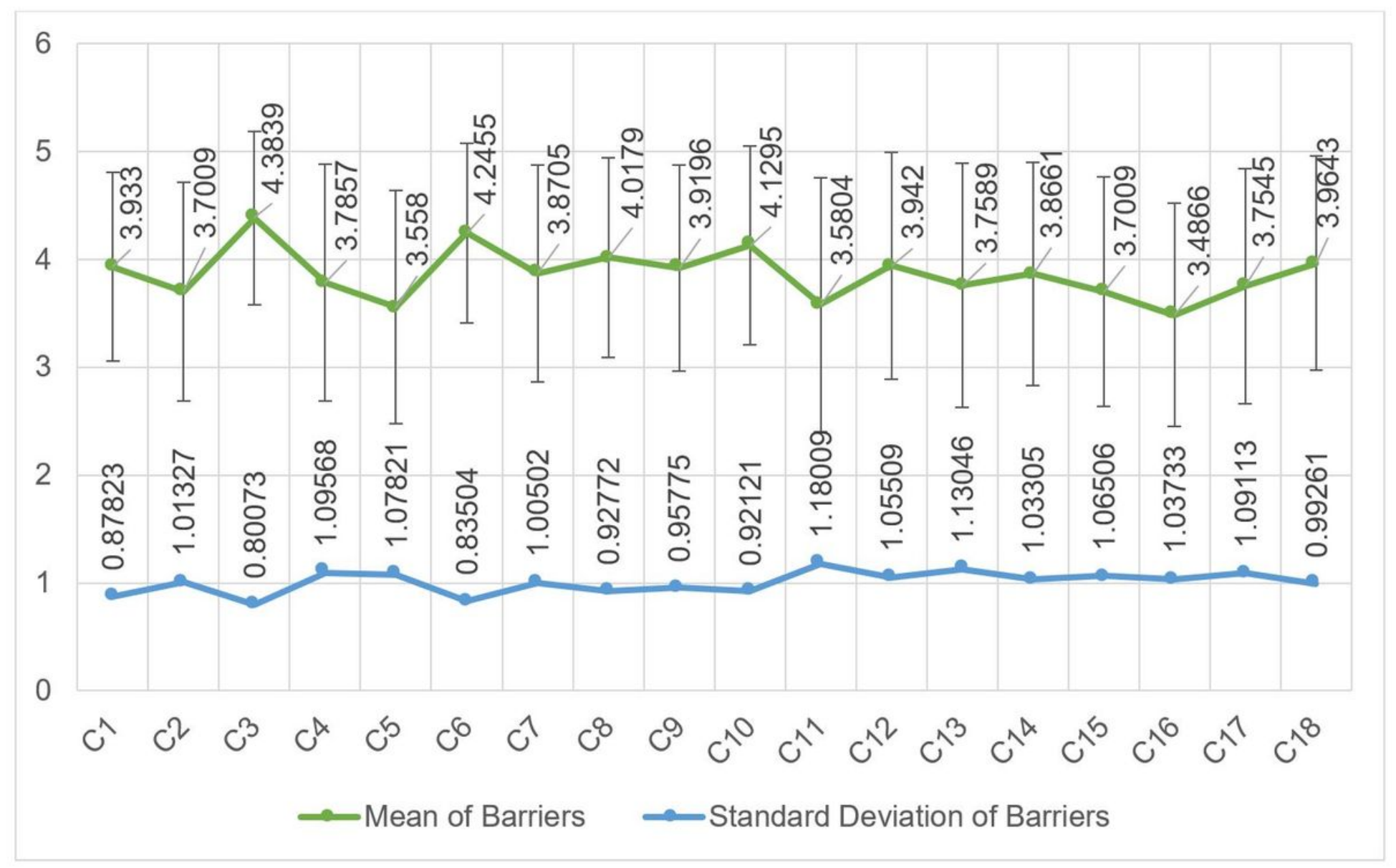

Figure 9

Descriptive statistics analysis for Barriers 


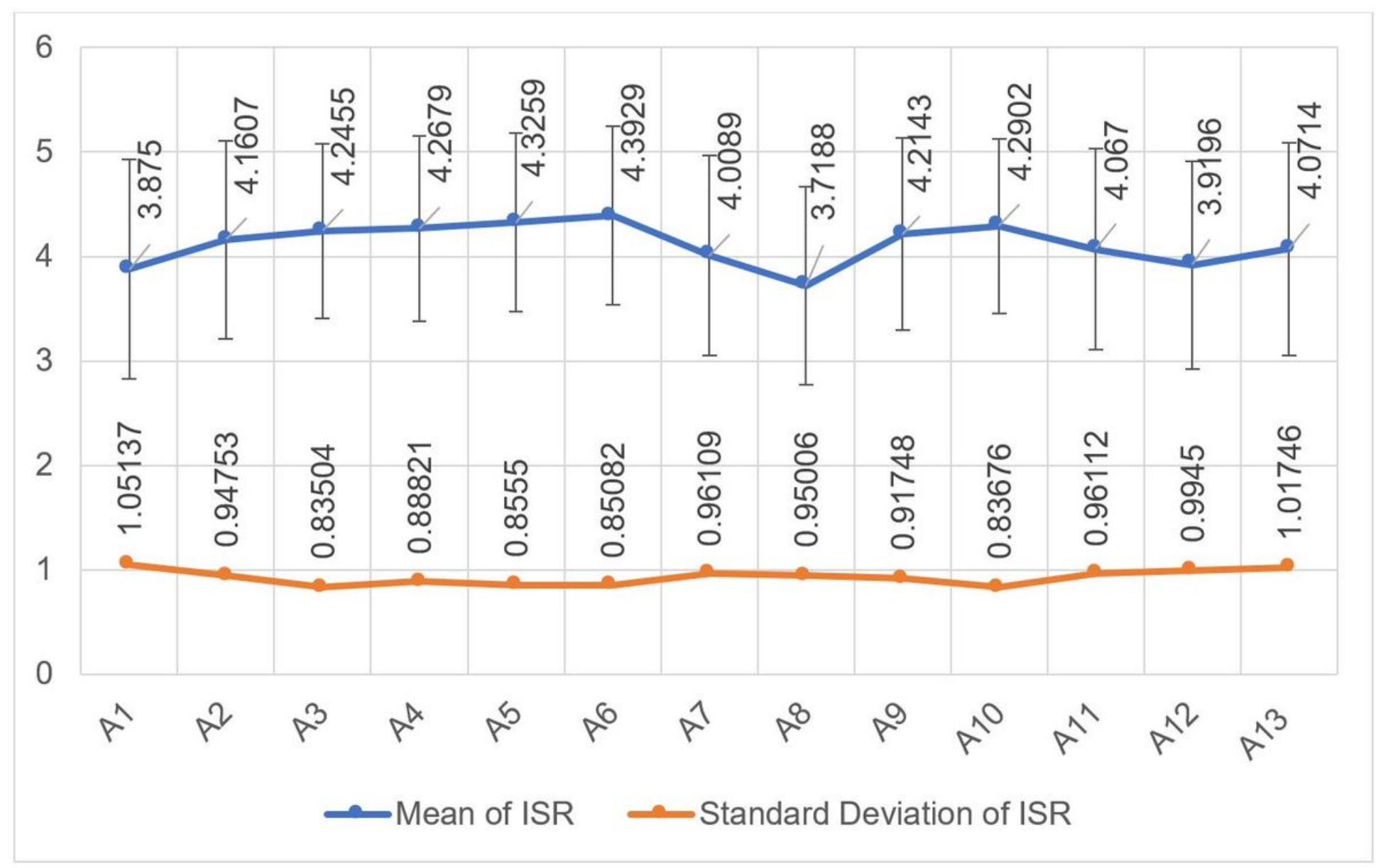

Figure 10

Descriptive statistics analysis for ISR 